\title{
Revealing Correlations Between Size Polydispersity and Deformability of Idealized Granular Materials using DEM
}

\section{Jizhong He}

Engineering Computing Center, Beijing Urban Construction Design and Development Group Co., Limited Yuzhen Han ( $\square$ hanyuzhen@bjucd.com )

Engineering Computing Center, Beijing Urban Construction Design and Development Group Co., Limited Lei Zhang

Beijing Urban Construction Group Co., Ltd.

\section{Gang Deng}

State Key Laboratory of Simulation and Regulation of Water Cycle in River Basin, China Institute of Water Resources and Hydropower Research

\section{Research Article}

Keywords: granular materials, deformability, particle size polydispersity, the distinct element method, micromechanical properties

Posted Date: February 9th, 2022

DOI: https://doi.org/10.21203/rs.3.rs-1291542/v1

License: (1) This work is licensed under a Creative Commons Attribution 4.0 International License. Read Full License 
Revealing Correlations Between Size Polydispersity and

Deformability of Idealized Granular Materials using DEM

\author{
Jizhong $\mathrm{He}^{1, \dagger}$, Yuzhen Han $^{1, *}$, Lei Zhang ${ }^{2}$, Gang Deng ${ }^{3}$
}

${ }^{1}$ Engineering Computing Center, Beijing Urban Construction Design and

Development Group Co., Limited, Beijing 100034, China

${ }^{2}$ Beijing Urban Construction Group Co., Ltd., Beijing 100088, China

${ }^{3}$ State Key Laboratory of Simulation and Regulation of Water Cycle in River Basin,

China Institute of Water Resources and Hydropower Research, Beijing 100048, China

† ORCID: 0000-0001-8586-7962

* Corresponding author: hanyuzhen@bjucd.com 


\section{Abstract}

This paper numerically investigates the deformability of granular materials with emphasis on the influence of particle size polydispersity. Triaxial compression tests are simulated using distinct element method (DEM) on a set of samples consisting of uncrushable spheres following a variety of grain size distributions. The compressibility under isotropic compression and deformation modulus during triaxial shearing are examined to correlate with the size polydispersity. The results show that, given the same relative density, the compressibility and deformation modulus is insensitive to the grain-size conditions under consideration, but the void ratio is significantly influenced by the size polydispersity. A grading index proposed by the authors is employed to quantify the size polydispersity and correlate with the deformation parameters. Compared with the more conventional coefficient of uniformity, this grading index exhibits a better performance for faithfully representing size polydispersity. An empirical prediction model for the deformation modulus is developed to account for the effect of the size polydispersity, in addition to the confining pressure and void ratio. Although this model exhibits a fair performance for predicting the deformation modulus, it is considered promising in accounting for the effect of size polydispersity. At the microscale, a unique correlation is found between the deformation modulus and the mechanical average coordination number, which can be estimated from the effective void ratio.

Keywords: granular materials; deformability; particle size polydispersity; the distinct element method; micromechanical properties 


\section{Introduction}

Size-polydisperse granular materials (i.e. composed of particles with different sizes) are commonly used in the field of geotechnical and geological engineering to construct earthworks such as rockfill dams and railway track blasts. Deformation parameters of these materials are vital to precisely predict the deformation of the earthworks they form. In recent decades, many investigations have been performed on the deformability of granular materials. It was concluded that the deformation behavior of granular materials is usually complex, highly dependent on the grain-scale characteristics of particles (e.g. particle shape and size) that constitute the materials, in addition to the confining pressure, packing density, and loading path [1-5]. Progressively increasing research on granular materials has focused on the effect of the grain-scale attributes on their macroscopic deformation response. Of particular interest for size-polydisperse granular materials is the relationship between size polydispersity, referring to heterogeneity of particle sizes in a mixture, and their macroscopic deformation parameters.

Researchers investigate the influence of size polydispersity by varying the grain size distribution (GSD) of granular materials. Many experiments have been conducted to establish correlations between deformation properties of granular materials and their GSDs. However, those studies did not necessarily reach consensus, mainly due to two underlying difficulties. First, gradation is usually not the only difference between the materials. Inevitable differences among them in grain-scale characteristics (e.g. shape and crush resistance of grains) sometimes give rise to contradictory results. For example, using the bender element technique, Bartake and Singh [1] found that the small-strain shear modulus of quartz sands increased as the mean particle size $D_{50}$ decreased, whereas Sharifipour et al. [6] reported this modulus of glass beads increased 
with increasing $D_{50}$. Second, data interpretation is to a great extent dependent upon selected measures of size polydispersity in individual studies. To develop practicallyuseful correlations with deformation properties, the mean particle size $D_{50}$ and the coefficient of uniformity $C_{u}=d_{60} / d_{10}$ (where $d_{60}$ and $d_{10}$ represent the diameters respectively corresponding to $60 \%$ and $10 \%$ finer) are commonly employed as main quantifiers of size polydispersity. However, the correlation with either alone cannot fully capture the gradation-dependence. For instance, Wichtmann and Triantafyllidis [7] concluded that the small-strain shear modulus was significantly dependent on $C_{u}$ but independent of $D_{50}$, while Liu et al. [8] showed that both $C_{u}$ and $D_{50}$ had pronounced effects on the small-strain shear modulus. These contradictory findings led to different empirical prediction models for the small-strain shear modulus, suggesting that the $D_{50}$ and $C_{u}$, derived respectively from only one and two points on a GSD curve, is too crude to characterize size polydispersity. Moreover, in some cases where the GSD is highly truncated $[9,10], d_{10}$ or even $d_{60}$ can often not be determined and $C_{u}$ cannot be calculated. Although some other descriptors are developed to better characterize size polydispersity, such as the moments of GSD [11, 12], and the breakage potential defined from the area under a GSD curve [13, 14], their validity in the gradationdependent correlations requires further investigations.

With the capability of precisely controlling grain-scale properties, the distinct element method (DEM) [15] appears as a suitable and powerful tool to investigate gradation-dependent behavior of materials. Numerical investigations were performed to reveal the influence of size polydispersity on some aspects of deformation response of granular materials, such as small strain stiffness [16], one dimensional compression $[10,17]$, and triaxial (or biaxial) compression [9, 12, 18]. Gu et al. [16] observed that, at the same confining pressure and void ratio, the small strain shear modulus decreased 
with increasing $C_{u}$. Minh and Cheng [10] found that the better-graded materials are less compressible than the more uniformly-graded materials. Note that in these studies, only qualitative relations were identified between the deformation parameters and size polydispersity, and additional efforts are needed to establish the quantitative ones. The authors of the current paper using biaxial compression simulations demonstrated that the deformation behavior of granular materials was affected by not only the spread but also the shape of the GSD curve, and proposed two grading indexes to correlate with the deformation parameters [12]. Nonetheless, the behaviors of granular materials under two-dimensional (2D) deformation conditions are very far from those of realistic materials, indicating that the developed correlations in [12] were also qualitative. The further extension of those achievements to three-dimensional (3D) models is worthwhile and essential for quantitatively linking the deformation parameters to size polydispersity.

This paper aims at a quantitative investigation on the relationship between size polydispersity of granular materials and their deformation parameters using 3D DEM simulations. The simulation program is composed of a series of drained triaxial compression tests on size-polydisperse granular materials featuring different GSDs (varying in both the spread and the shape). In this preliminary phase, we use uncrushable spheres to represent the constituent particles. The idealized materials allow the effects of other grain-scale properties (e.g. particle shape and breakage) to be isolated, and therefore any observed difference is attributable to the difference in size polydispersity. Nevertheless, combined effects of size polydispersity, particle shape and breakage can be introduced successively in further studies. The previously proposed grading index [12] is compared with the conventional $C_{u}$ to validate its better performance in characterizing size polydispersity and thus gradation-dependent 
correlations. Moreover, attempts are also made to seek microscopic variables that govern deformation behavior of granular aggregates as GSD varies.

This paper is organized as follows: Section 2 introduces the numerical method employed in this study and model configuration. Section 3 briefly introduces the definition of the previously developed measure of size polydispersity to be used in the subsequent analyses. Section 4 presents the simulation results based on which gradation-dependent correlations are established for deformation parameters through the adopted measures of size polydispersity. The main conclusions drawn from this study are given in Section 5 .

\section{DEM simulations}

\subsection{Characterizing GSD}

We characterize a GSD curve in two aspects: the spread and the shape. The procedures of the characterization were detailed in the previous publication [12]. The spread of the GSD is described by a dimensionless indicator, i.e., the size span $s=\left(d_{\max }-d_{\min }\right) /\left(d_{\max }\right.$ $+d_{\min }$ ), in which $d_{\max }$ and $d_{\min }$ denote the largest and smallest particle diameters, respectively. Note that the case $s=0$ represents a monodisperse system composed of equal-size particles, whereas $s=1$ corresponds to an "infinitely" polydisperse distribution. The shape of the GSD curve is described by introducing the cumulative $\beta$ distribution:

$$
\beta(x)=\frac{\Gamma(a+b)}{\Gamma(a) \Gamma(b)} \int_{0}^{x} t^{a-1}(1-t)^{b-1} \mathrm{~d} t
$$


where $\Gamma(\cdot)$ is the Gamma function; and positive parameters $a$ and $b$ control the shape of the distribution. The GSD with the particle diameters $d$ over the interval $\left[d_{\max }, d_{\min }\right]$ is then modelled as follows:

$$
P(d)=\beta\left(d_{\mathrm{r}}\right) \times 100 \%, d_{\mathrm{r}}=\frac{d-d_{\min }}{d_{\max }-d_{\min }}
$$

where $P(d)$ is the percentage in mass of particles finer than $d ; d_{\mathrm{r}} \in[0,1]$ is the reduced (dimensionless) diameter. This model allows for approximating various GSDs typically occurring in soils (e.g. linear, power-law and S-shaped distributions) by varying the values of $s, a$ and $b[19,20]$.

In the simulations presented in this paper, we fix $d_{\min }=3 \mathrm{~mm}$ and $a=2$ while vary $s$ and $b$ to obtain a range of gradation with various spreads and shapes. The parameters $d_{\min }$ and $a$ is set arbitrarily considering that the simulation results is primarily influenced by the relative particle size (not the absolute size). The size span is varied in the following range: $s \in[0.40,0.54,0.89]$. For $s$ being 0.40 or 0.54 , the parameter $b$ varies from 0.1 or 0.5 (a power-law distribution in the semi-log representation) to 4 (a Sshaped GSD curve in the same coordinate frame). To ensure statistical representation of a sample, its total number of particles $N$ should be sufficiently large; in general, the larger $s$ and $b$ of a GSD requires more particles. Accordingly, the value of $b$ for $s=0.89$ is only 0.5 due to the limitation of $N$ and hence computational cost. The procedure for numerically generating a set of particles following a prescribed GSD curve was specified by He et al. [12]. By trial and error, 10,000 particles for $s=0.40$ and 0.54, and 24,000 particles for $s=0.89$ are chosen to obtain a good fit to theoretical GSDs with affordable computational efforts. Figure 1 shows the GSD curves of ten numerical 
samples, and the generated GSDs (denoted with symbols) coincide well with the corresponding theoretical ones (denoted with solid lines).

\subsection{The simulator and the inter-particle contact law}

In this study, the open-source DEM code YADE is employed to perform the numerical simulations [21]. A simple linear force-displacement contact model in conjunction with Coulomb's friction criterion is adopted to describe the inter-particle contact. This interparticle contact law requires three input parameters: Young's modulus $E_{c}$, tangentialto-normal stiffness ratio $\theta$ and inter-particle friction coefficient $\mu$, through which the normal and tangential stiffnesses between two contacting particles with radii $r_{\mathrm{a}}$ and $r_{\mathrm{b}}$ are determined as follows:

$$
k_{n}=\frac{2 E_{c} r_{\mathrm{a}} r_{\mathrm{b}}}{\left(r_{\mathrm{a}}+r_{\mathrm{b}}\right)}
$$

$$
k_{s}=\theta k_{n}
$$

To ensure quasi-static loading, we add a simple local non-viscous damping force to dissipate kinematic energy [22]:

$$
f^{\text {damp }}=\alpha f^{\text {resid }}
$$

where $\alpha$ represents the damping ratio, and $f^{\text {resid }}$ is the magnitude of the residual force accumulated from all the contact forces on a particle. The direction of the damping force is opposite to the velocity of the particle.

The model parameters used in the simulations are tabulated in Table 1. Published studies have demonstrated that the lower the particles' stiffness, the larger the contact overlap between two particles [23, 24]. To alleviate the impact of the contact overlaps 
on the deformation behavior of granular aggregates, the Young's modulus of particles $E_{c}$ is chosen about one order of magnitude larger than those reported in previous DEM simulations $[18,25-27]$. The stiffness ratio $\theta$ is set as 0.7 , within a suggested range of realistic granular materials [28]. Local non-viscous damping ratio $\alpha=0.2$ is considered to avoid over-damping [12]. The inter-particle friction coefficient $\mu=0.7$, corresponding to a friction angle of $35^{\circ}$, is chosen slightly higher than those for some common minerals measured between smooth surfaces [29]. This higher value to some extent captures the effect of particles' surface roughness.

\subsection{The simulation procedure}

The DEM simulations are performed under gravity-free conditions in two steps: (1) sample preparation, and (2) drained triaxial compression loading. During sample preparation, non-contacting particles with random initial coordinates are generated according to a pre-defined GSD within a cubical periodic cell [30, 31]. The resulting assembly are then isotropically compressed at $10 \mathrm{kPa}$ by moving all the boundaries of the cell with a stress-controlled servo mechanism. In this study, each assembly with a given GSD is compressed to two states: loose state and dense state, which here are used in a relative sense. To obtain a loose sample, the inter-particle coefficient of friction is set to 1.0 and particle rotations are switched off until the mean stress reaches $90 \%$ of the target value. Then, the inter-particle friction coefficient is changed to 0.7 and particle rotations are switched on. On the basis of the loose sample, a dense sample is attained by altering the inter-particle friction coefficient into 0 and maintaining $10 \mathrm{kPa}$ confining pressure, and eventually resetting the inter-particle friction coefficient to 0.7 . When the ratio of the maximum unbalance force to the mean contact force is smaller than a pre-defined tolerance, $10^{-3}$ according to $[32,33]$, we assume that the system 
reaches force equilibrium and the sample preparation phase ends. This standardized procedure suggests that the produced loose (or dense) samples with various GSDs are at a similar level of relative density. The initial void ratios $e_{0}$ are listed in Table 2 along with other descriptors of the resulting samples. $e_{0}^{\max }$ and $e_{0}^{\min }$, namely the maximum and minimum initial void ratios, are respectively measured from the loose and dense samples. Figure 2 exemplifies the resultant samples at different density states. The minimum ratio of the sample dimension to the largest particle diameter of all samples is larger than 10 , which is the minimum recommended value to limit the effect of boundary conditions $[34,35]$.

To examine the homogeneity of the samples, the void ratio distribution inside them is determined in a careful manner. According to [36], the volume of every sample is divided into six isochoric slices extending throughout the whole sample in each direction, i.e., $\mathrm{X}, \mathrm{Y}$ and $\mathrm{Z}$ directions in Fig. 2. In our simulations, the thickness of each slice is larger than twice the maximum particle diameter. The void ratio within a given slice $i$ is calculated as follows:

$$
e_{i}=\left(V_{i}^{\mathrm{s}}-V_{i}^{\mathrm{p}}\right) / V_{i}^{\mathrm{p}}
$$

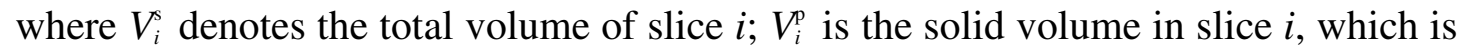
the sum of the volume of fully-enclosed particles and that of intersected boundary particles whose fractions outside the zone are subtracted. Figure 3 presents the distributions of void ratio in six samples (similar in other unpresented samples), suggesting adequate homogeneity in these samples since the largest deviation of $e_{i}$ from the void ratio of the whole sample is smaller than 5\%. Meanwhile, the void ratio distribution in a sample under dense conditions is more even than that under loose conditions. 
The drained triaxial compression simulation encompasses an isotropic compression phase followed by a shear loading phase. Every sample is isotropically compressed at a confining pressure ranging from 300 to $900 \mathrm{kPa}$ until force equilibrium is reached. Afterwards, the top and bottom boundaries of the sample are vertically (i.e. Ydirectionally) moved at a constant strain rate of $0.04 / \mathrm{s}$ to exert shear loading. This loading rate is chosen to ensure quasi-static conditions inferred from the resulting inertia number defined by da Cruz et al. [37] being no more than $10^{-3}$ [38]. During shearing, the confining pressure on the side boundaries is maintained constant by servocontrol. This shearing process of all samples terminates as an axial strain of $20 \%$ is reached. The axial strain $\varepsilon_{1}$ is computed from the boundary movements:

$$
\varepsilon_{1}=\frac{h_{0}-h_{1}}{h_{0}}
$$

where $h_{0}$ and $h_{1}$ respectively denote the initial and current heights (i.e. paralleling with the axial direction) of the sample. The well-known Love-Weber formula [39, 40] is introduced to calculate the macro-scale homogenized stress tensor of a granular aggregate; and the deviatoric stress $q$ is derived from the major principal stress $\sigma_{1}$ (in the axial direction) and the minor principal stress $\sigma_{3}$ (in the lateral direction), i.e., $q=$ $\sigma_{1}-\sigma_{3}$

To normalize the contact overlap, the overlap ratio is defined as follows:

$$
\chi=\frac{u_{n}}{4 r_{\mathrm{a}} r_{\mathrm{b}} /\left(r_{\mathrm{a}}+r_{\mathrm{b}}\right)}
$$

where $u_{n}$ represents the normal overlap distance between two contacting particles with radii $r_{\mathrm{a}}$ and $r_{\mathrm{b}}$. Throughout these simulations, the maximum value of the overlap ratio 
is less than 5\%, which proves the chosen particle stiffness is sufficient for resisting contact overlapping $[10,41]$.

\section{Measures of size polydispersity}

The straightforward and widely-used descriptors of size polydispersity, such as the size spread $\left(d_{\max }-d_{\min }\right)$ and the coefficient of uniformity $C_{u}$, are considered not representative of the entire GSD due to their derivation from a limited number of points on a GSD curve. To faithfully represent size polydispersity of a granular mixture, we considered the combined effect of the spread (i.e. the size span $s$ ) and the shape of a GSD curve, and defined a grading index $I_{G}$ in previous work [12]:

$$
I_{G}=s \gamma ; \gamma=\sqrt{\sum_{i=1}^{N}\left(d_{\mathrm{r}}^{i}\right)^{2} w_{\mathrm{m}}^{i}-\left(\sum_{i=1}^{N} d_{\mathrm{r}}^{i} w_{\mathrm{m}}^{i}\right)^{2}}
$$

where $d_{\mathrm{r}}^{i}$ denotes the reduced diameter of the $i^{\text {th }}$ particle; $w_{\mathrm{m}}^{i}$ denotes the mass percentage of the $i^{\text {th }}$ particle to the total mass of the mixture; and $\gamma$, the grading shape index, is the standard deviation of the reduced diameters weighted by their corresponding mass percentage. This summation form of $\gamma$ poses a practical difficulty in the application of $I_{G}$ due to its requirement for knowledge of sizes of individual particles. To improve the applicability of $I_{G}$, we derived the integral form of $\gamma$ for a mixture with continuous gradation [12]. The coefficient of uniformity $C_{u}$, grading shape index $\gamma$ and grading index $I_{G}$ evaluated from the GSDs in this study are listed in Table 2.

\section{Results}

The simulations of drained triaxial compression tests are conducted, and the results obtained from the isotropic compression phase and the shear loading phase are separately analyzed. Taking the advantage of DEM in providing grain-scale insights, 
we explain the observed deformation behavior of the simulated granular materials from a microscopic perspective.

\subsection{Isotropic compression}

Figure 4 plots the void ratios $(e)$ at the end of isotropic compression stage against the confining pressures $\left(\sigma_{3}\right)$, i.e., the isotropic compression curve. For all samples, the void ratio decreases linearly with the increase of the confining pressure, which is consistent with the observations from previous 3D DEM simulations of isotropic compression tests $[9,42]$. This linear $e-\sigma_{3}$ relation suggests that the isotropic compression behavior of a granular mixture can be approximately characterized by its initial void ratio $\left(e_{0}\right)$ and compressibility (i.e. the slope of the isotropic compression curve). As shown in Fig. 4 , the $e-\sigma_{3}$ curves of samples at a similar relative density are nearly parallel to each other despite different GSDs; and compared to loose samples (see Fig. 4(a)), those of dense samples (see Fig. 4(b)) are apparently flatter. It indicates that the uncrushable spherical granular packing at a higher relative density exhibits higher resistance to compaction and its compressibility is negligibly affected by the size polydispersity under consideration. Unlike the gradation-independent compressibility, the initial void ratio $\left(e_{0}\right)$ is notably dependent on both the spread and the shape of GSD. Figure 5 presents $e_{0}$ against the size span $s$ and the grading shape index $\gamma$. It can be observed that, for samples under the same packing conditions (i.e. the same confining pressure and relative density), the initial void ratio $\left(e_{0}\right)$ decreases as the two parameters increase, although few exceptions occur as $s=0.54$. This figure indicates that a sample with higher size polydispersity (i.e. with larger $s$ and $\gamma$ ) tends to be denser under a specific pressure. Figure 6 plots $e_{0}$ against different measures of size polydispersity. It is noted that $e_{0}$ linearly decreases with the degree of size polydispersity, quantified by either $C_{u}$ 
or $I_{G}$, for samples under similar packing conditions. The better linear approximation for $e_{0}^{\min }$ than for $e_{0}^{\max }$ is probably attributable to a more stable quality control in generating dense samples and hence more consistent packing conditions (see Fig. 3). As illustrated in Fig. 6, the difference between $I_{G}$ and $C_{u}$ in correlating $e_{0}$ is insubstantial despite a slightly higher value of coefficient of determination $\left(R^{2}\right)$ from $I_{G}$. This could be attributed to the low degree of size polydispersity (e.g. values of $C_{u}$ being smaller than 2) of the GSDs in present study [12]. To validate and broaden the applicability of $I_{G}$, it is utilized to approximate the $e_{0}$-data from the literature. Figure 7 collects $e_{0}$ measured in the laboratory on blast beads [43] and in the DEM simulation on idealized granular aggregates $[9,10]$. Figure 7(a) displays $e_{0}$ of blast beads against $I_{G}$ and $C_{u}$ [43]. A poor correlation between $e_{0}$ and $C_{u}$ is observed since the GSDs with an identical $C_{u}$ but various $D_{50}$ cannot be well distinguished. Compared with $C_{u}, I_{G}$ performs much better at approximating $e_{0}$, implying that $I_{G}$ is able to more precisely represent the GSD, i.e., better capture the size polydispersity, of a mixture. Figure $7(\mathrm{~b})$ shows the relation between $e_{0}$ and different descriptors of size polydispersity from idealized granular aggregates $[9,10]$. The mass fraction of small particles is employed to characterize size polydispersity since the GSDs is highly truncated and therefore cannot be evaluated with commonly-used descriptors, such as $C_{u}$ and $D_{50}$. As shown in Fig. 7(b), the adoption of $I_{G}$ results in the collapse of data on a master line, providing evidence that $I_{G}$ is a promising descriptor for flexibly and effectively representing size polydispersity of highly truncated GSDs.

\subsection{Triaxial shear}

\subsubsection{Stress-strain response}


Figure 8 and Figure 9 show the deviatoric stress-strain response obtained respectively from loose and dense samples with various GSDs under different confining pressures. With the DEM model and selected parameters, the simulations are able to produce reasonable results qualitatively in good accordance with experimental observations from granular materials under triaxial compression conditions [44, 45]. Despite the variation in the confining pressure, all loose and dense samples respectively exhibit typical strain-hardening and strain-softening behavior. As the axial strain develops, the deviatoric stress of dense samples increases up to a peak and then decreases to a relatively stable value, while that of loose samples increases until a stable value is reached. Higher the confining pressure, higher the peak and stable deviatoric stresses are reached. As shown in Fig. 8 and Fig. 9, under similar packing conditions, negligible differences among the stress-strain responses, particularly at the initial stage, of samples with various GSDs are observed. This implies that the stiffness of the packing of uncrushable spheres is independent of size polydispersity.

\subsubsection{Correlations for deformation modulus}

The deformation modulus $E_{50}$, defined as the secant slope of the stress-strain curve when the deviatoric stress equals half of the maximum strength, is often employed in earth structure design for deformation calculations [12]. The values of $E_{50}$ in this work are obtained from the deviatoric stress-strain curves of the triaxial compression tests. It is worth mentioning that the maximum deviatoric stress of loose samples is averaged for $\varepsilon_{1}$ ranging from $16 \%$ to $20 \%$ to smooth inevitable scatter of data. Figure 10 presents $E_{50}$ from both dense and loose samples under different confining pressures. In good agreement with the well-recognized pressure- and density-dependence of deformability $[46,47], E_{50}$ increases with increasing confining pressure and density for all samples. 
Note that as the density decreases, the sensitivity of $E_{50}$ to the confining pressure increases. This indicates that the microstructure of a loose packing changes more remarkably with increasing confining pressure than that of a dense packing. In general, given the same packing conditions, the data from samples with various GSDs collapse in a narrow range, confirming that the stiffness is independent of size polydispersity.

Recognizing that relative density is practically a rather weak parameter due to the difficulty in determining maximum and minimum void ratio [48, 49], researchers are often inclined to adopt void ratio as an index of the deformation characteristics of granular materials $[7,8,50,51]$. To illustrate the effect of size polydispersity, $E_{50}$ of samples with various GSDs is plotted versus the void ratio $e$ in Fig. 11. Figure 11 reveals that, given a specific confining pressure, the $E_{50}-e$ curve shifts left as $I_{G}$ (i.e. the degree of size polydispersity) increases. This means that, at the same void ratio and confining pressure, the deformation modulus $E_{50}$ decreases with increasing size polydispersity; the phenomenon agrees well with the experimental observations where size polydispersity was represented by $C_{u}$ [47]. Thus, while considering a constant relative density there is a negligible influence of the size polydispersity on the deformation modulus $E_{50}$, this influence is significant when a constant void ratio is taken as the basis of comparison. This finding can be explained by the fact that the two extremities of void ratio of a granular aggregate decrease with increasing size polydispersity (see Fig. 6). Therefore, given a constant void ratio, a higher degree of size polydispersity means a lower relative density, leading to a smaller value of $E_{50}$.

Inspired by the well-known empirical formula for the small strain shear modulus $[52,53]$, we develop a prediction model for $E_{50}$ in function of void ratio $e$ and confining pressure $\sigma_{3}$ : 


$$
E_{50}=A \times f(e) \times\left(\frac{\sigma_{3}}{p_{\mathrm{a}}}\right)^{n} ; f(e)=e^{-m}
$$

where $f(e)$ is a function of void ratio $e$ with a power-law form proposed by Lo Presti et al. [54]; $p_{\mathrm{a}}=100 \mathrm{kPa}$ represents the reference atmospheric pressure; $A, n$ and $m$ are three model parameters. To account for size polydispersity, the three model parameters are tentatively assumed as the functions of $I_{G}$ and correlations between them are developed. First, as shown in Fig. 11, the parameter $m$ is determined for a certain confining pressure by fitting the data with a function of the form $E_{50}(e)=k f(e)$ (the constant $k$ is not used further). Figure 12 plots the parameter $m$ versus $I_{G}$. For each sample the three values for the three confining pressures are given. Despite remarkable scatter of data, the parameter $m$ has a tendency to decrease with increasing $I_{G}$, and this tendency may be described by the exponential function:

$$
m=c_{1} \exp \left(-c_{2} I_{G}\right)
$$

with constants $c_{1}=6.045$ and $c_{2}=0.492$ (see Fig. 12). To determine the parameters $A$ and $n$, the deformation modulus $E_{50}$ is afterwards divided by the void ratio function $f(e)$, and the normalized deformation modulus $E_{50} / f(e)$ is plotted against confining pressure $\sigma_{3}$. Figure 13 shows three examples for determining the values of parameters $A$ and $n$ according to Eq. (10). The obtained values of $A$ and $n$ are plotted against $I_{G}$ in Fig. 14(a) and (b), respectively. It is observed in Fig. 14(a) that parameter $A$ decreases with increasing $I_{G}$ and this relationship can be approximated by a function composed of a constant and a power-law portion:

$$
A=c_{3}-c_{4} I_{G}^{c_{5}}
$$


with constants $c_{3}=8.798, c_{4}=30.909$, and $c_{5}=1.036$. Figure $14(\mathrm{~b})$ shows that parameter $n$, by and large, increases with $I_{G}$ despite appreciable scatter of data. It may be expressed by the power-law function:

$$
n=c_{6} I_{G}^{c_{7}}
$$

with constants $c_{6}=0.389$ and $c_{7}=0.119$. On the basis of the model parameters obtained from Eqs. (11), (12) and (13), a predictive $E_{50}$ model which accounts for the effect of size polydispersity can be derived, yielding a relationship as follows:

$$
E_{50}=\left(8.798-30.909 I_{G}^{1.036}\right) \times e^{-6.045 \exp \left(-0.492 I_{G}\right)} \times\left(\frac{\sigma_{3}}{p_{\mathrm{a}}}\right)^{0.389 I_{G}^{0.19}}
$$

For comparison, the model parameters in Eq. (10) are also correlated with $C_{u}$ by the same workflow. In this way, an alternative empirical expression for $E_{50}$ where size polydispersity is represented by $C_{u}$ can be acquired as follows:

$$
E_{50}=\left(7.369-0.310 C_{u}^{4.091}\right) \times e^{-6.809 \exp \left(-0.118 C_{u}\right)} \times\left(\frac{\sigma_{3}}{p_{\mathrm{a}}}\right)^{0.269 C_{u}^{0.262}}
$$

Figure 15 presents the $E_{50}$-values predicted either by Eq. (14) or Eq. (15) as a function of the measured values. It can be observed that the two models yield approximately the same predictions, and the data cluster around the bisecting line falls within a relative error range of $\pm 25 \%$. The similar predictive results suggest a negligible difference between $I_{G}$ and $C_{u}$ in characterizing size polydispersity, which is probably attributable to the low degree of size polydispersity of the GSDs in this study. The relatively large prediction errors may be due to a limited applicability of the void ratio function $f(e)$. Note in Fig. 12 that parameter $m$ decreases with increasing confining pressure and the best-fit curve basically runs through the points obtain from samples 
under $600 \mathrm{kPa}$ confining pressure. The underestimation or overestimation of $m$ values would respectively give rise to an increase or decrease of the normalized deformation modulus $E_{50} / f(e)$, i.e., an increase of $E_{50} / f(e)$ for samples under $300 \mathrm{kPa}$ confining pressure and a decrease of that under $900 \mathrm{kPa}$ confining pressure. The smaller void ratio $e$ of dense samples (i.e. a smaller base of the void ratio function) leads to more dramatic changes of $E_{50} / f(e)$, and therefore the decrease of $E_{50} / f(e)$ with increasing confining pressure in Fig. 13. It can be observed in Fig. 13 that the best-fit relationships significantly underestimate $E_{50} / f(e)$ for samples at the dense state and $300 \mathrm{kPa}$ confining pressure or at the loose state and $900 \mathrm{kPa}$ confining pressure; and for samples at the loose state and $300 \mathrm{kPa}$ confining pressure or at the dense state and $900 \mathrm{kPa}$ confining pressure, $E_{50} / f(e)$ is noticeably overestimated. Those inaccurate estimations are mainly originated from the pressure-independence of the void ratio function $f(e)$, and are in good concert with the prediction errors in Fig. 15. Despite the limited predictive performance, the proposed model exhibits a promising capability of accounting for size polydispersity. Meanwhile, the model is developed on the basis of limited data due to the prohibitive computational cost, and further refinement and verification of this empirical method are worthwhile when an enriched data set becomes available.

\subsection{Micromechanical explanation of the observations}

The fabric of a particulate system, referring to the particles' spatial configuration in this study, proves to have considerable effects on its macroscopic mechanical behaviors. To quantitatively characterize the fabric of a granular sample, the average coordination number $(\mathrm{CN})$, i.e. the average number of contact(s) of individual particles, is introduced. Generally, higher $\mathrm{CN}$ means more neighbor particles in average, and therefore higher stability of the sample. Informed with this basic knowledge, we plot 
the deformation modulus $E_{50}$ with $\mathrm{CN}$ of the samples before shearing. As illustrated in Fig. 16, a one-on-one relation between the two variables can be established only if GSD remains constant, implying that $\mathrm{CN}$ fails to uniquely determine $E_{50}$. Additionally, with increasing $I_{G}$, the decrease in both $\mathrm{CN}$ and void ratio $e$ (see Fig. 11) is counter-intuitive since a positive correlation is normally considered between the packing density and $\mathrm{CN}$ of granular aggregates.

To explore the gradation-dependent fabric, the number of contact(s) per particle in the dense samples before loading is statistically analyzed. Figure 17 shows the resulting distributions of contacts and the percentage of particles with 20 contacts covers that of particles with more than 20 contacts. Note that as $I_{G}$ increases, the percentage of particles without any contact (i.e. floating particles) increases. This increasing percentage of floating particles gives a reason to the decrease of CN in Fig. 16, although they increase the density (i.e. decrease the void ratio) at the macroscale. It is interesting that the percentage of particles with 1 3 contacts in these numerical samples is quite small. This observation corroborates the findings of Taiebat et al. [55] that a local configuration in which a particle is stuck by at least 4 neighboring particles is stable. For the particles with no less than 4 contacts, the curves shift left and the peak contact number decreases as $I_{G}$ increases, suggesting a less uniform distribution of contacts in a more polydisperse sample.

The mechanical average coordination number $(\mathrm{MCN})$ is defined by Thornton [31] as the average coordination number only counting particles with more than one contact that contribute to the loading frame. To exclude the influence of floating particles on the macro- and microscopic relations, we attempt to correlate $E_{50}$ with $\mathrm{MCN}$ of the samples before shearing. As illustrated in Fig. 18, the data collapse in a narrow band, 
implying that $\mathrm{MCN}$ succeeds in uniquely determining $E_{50}$ at the particle level for the grain-size conditions under consideration.

The correlation in Fig. 18 could be useful in practice if MCN (which cannot be easily quantified in laboratory) can be estimated from conventionally measurable variables. Figure 19(a) shows an effort to link MCN with the void ratio $e$. A poor correlation is found due to the fact that $e$ decreases significantly but MCN changes slightly with increasing $I_{G}$. Considering the effect of floating particles, we define an effective void ratio $e^{\prime}$ that counts particles with no more than one contact as void volume. Figure 19(b) plots MCN against the effective void ratio $e^{\prime}$. A strong linear correlation between the two variables suggests that, although being practically unmeasurable, the effective void ratio $e^{\prime}$ is a more representative parameter for the deformation behavior of the granular materials under consideration.

\section{Conclusions}

In this paper, a series of drained triaxial compression tests are performed using 3D DEM on aggregates of uncrushable spheres to study the influence of particle size polydispersity on deformation behavior of granular materials. We characterize the size polydispersity of the materials from two aspects: the spread and the shape of GSD curves. From the simulation results, the following conclusions are drawn:

Under isotropic compression, the initial void ratio $e_{0}$ and compressibility are examined for various GSDs. The results show that, given the same relative density, the initial void ratio is significantly affected by both the spread and the shape of GSDs, but negligible differences in the compressibility are observed as the GSD varies. The negligible differences indicate that the compressibility of the idealized materials is insensitive to the grain-size conditions under consideration. Considering the combined 
effect of the spread and the shape of the GSD curve, a grading index $I_{G}$ previously proposed by the authors of the current paper is employed to quantify the size polydispersity, and it is well correlated with the initial void ratios. Compared with the more conventional coefficient of uniformity $C_{u}$, the grading index $I_{G}$ exhibits slight superiority in characterizing the GSDs in this study due to their low degrees of size polydispersity. However, since $I_{G}$ can well approximate the $e_{0}$-data from existing literature, where the GSDs cannot be clearly distinguished by $C_{u}$ or even derive $C_{u}$, it is considered as a promising descriptor for faithfully representing size polydispersity of various types of GSDs.

During triaxial shearing, the deformation modulus $E_{50}$ is measured to correlate with the size polydispersity. The results present that, while considering the same relative density, $E_{50}$ is negligibly influenced by the size polydispersity; when given a constant void ratio, $E_{50}$ decreases as the degree of the size polydispersity increases. This finding is explained by the fact that, given the same relative density, the void ratio decreases with the increasing degree of size polydispersity. Therefore, given a constant void ratio, higher degrees of size polydispersity implicate lower relative densities, leading to smaller values of $E_{50}$. An empirical prediction model for the $E_{50}$ is proposed to account for the effect of the size polydispersity, in addition to the confining pressure and void ratio. Although this model exhibits a fair performance for predicting the $E_{50}$, it is considered promising in accounting for the effect of size polydispersity. At the microscale, a unique correlation is found between the $E_{50}$ and the mechanical average coordination number regardless of variation in size polydispersity. Although being practically unmeasurable, this coordination number can be correlated to the effective void ratio. 
In this study, the degree of size polydispersity for all GSDs is quite low (e.g. values

of $\mathrm{Cu}$ are smaller than 2) due to the limitation of computational cost, whereas in practice, it is substantially higher. Further work is needed to check the suitability of the proposed model for granular materials within a wider range of size polydispersity.

\section{Data availability statements}

The datasets generated and/or analyzed during the current study are not publicly available due to the anonymity of the participants but are available from the corresponding author on reasonable request.

\section{Acknowledgements}

The work is supported by the Independent Research Fund of State Key Laboratory of Simulation and Regulation of Water Cycle in River Basin (with grant No. SKL2020ZY09), and the Science and Technology Project on Developing the Xiong'an New Area (with grant No. 2021-07 and 2021-03).

\section{References}

1. Bartake PP, Singh DN (2007) Studies on the determination of shear wave velocity in sands. Geomech Geoengin 2:41-49. https://doi.org/10.1080/17486020601065449

2. Enomoto T (2016) Effects of grading and particle characteristics on small strain properties of granular materials. Soils Found 56:745-750. https://doi.org/10.1016/j.sandf.2016.07.014

3. Patel A, Bartake PP, Singh DN (2009) An Empirical Relationship for Determining Shear Wave Velocity in Granular Materials Accounting for Grain Morphology. Geotech Test J 32:1-10. https://doi.org/10.1520/GTJ100796

4. Payan M, Khoshghalb A, Senetakis K, Khalili N (2016) Effect of particle shape and validity of $\mathrm{G}$ max models for sand: A critical review and a new expression. Comput Geotech 72:28-41. https://doi.org/10.1016/j.compgeo.2015.11.003

5. Payan M, Senetakis K, Khoshghalb A, Khalili N (2017) Effect of Gradation and Particle Shape on Small-Strain Young's Modulus and Poisson's Ratio of Sands. 
Int J Geomech 17:04016120. https://doi.org/10.1061/(ASCE)GM.19435622.0000811

6. Sharifipour M, Dano C, Hicher P-Y (2004) Wave velocities in assemblies of glass beads using bender-extender elements. Newark, DE, United States, p 9

7. Wichtmann T, Triantafyllidis T (2009) Influence of the Grain-Size Distribution Curve of Quartz Sand on the Small Strain Shear Modulus Gmax. J Geotech Geoenvironmental Eng 135:1404-1418. https://doi.org/10.1061/(ASCE)GT.1943-5606.0000096

8. Liu X, Zou D, Liu J, Zheng B (2021) Predicting the small strain shear modulus of coarse-grained soils. Soil Dyn Earthq Eng 141:106468. https://doi.org/10.1016/j.soildyn.2020.106468

9. He J, Liu F, Jiang M, et al (2018) Exploring Grading-Dependency of Deformation Modulus of Loose Aggregates of Spherical Particles Using DEM. In: Zhou A, Tao J, Gu X, Hu L (eds) Proceedings of GeoShanghai 2018 International Conference: Fundamentals of Soil Behaviours. Springer Singapore, Singapore, pp 826-834

10. Minh NH, Cheng YP (2013) A DEM investigation of the effect of particle-size distribution on one-dimensional compression. Géotechnique 63:44-53. https://doi.org/10.1680/geot.10.P.058

11. Guida G, Einav I, Marks B, Casini F (2020) Linking micro grainsize polydispersity to macro porosity. Int $\mathrm{J}$ Solids Struct 187:75-84. https://doi.org/10.1016/j.ijsolstr.2018.11.032

12. He J, Liu F, Deng G, Fu P (2021) Relationships between gradation and deformation behavior of dense granular materials: Role of high-order gradation characteristics. Int J Numer Anal Methods Geomech 45:1791-1808. https://doi.org/10.1002/nag.3224

13. Kikumoto M, Wood DM, Russell A (2010) Particle Crushing and Deformation Behaviour. Soils Found 50:547-563. https://doi.org/10.3208/sandf.50.547

14. Tengattini A, Das A, Einav I (2016) A constitutive modelling framework predicting critical state in sand undergoing crushing and dilation. Géotechnique 66:695-710. https://doi.org/10.1680/jgeot.14.P.164

15. Cundall PA, Strack ODL (1979) A discrete numerical model for granular assemblies. Géotechnique 29:47-65. https://doi.org/10.1680/geot.1979.29.1.47

16. Gu X, Lu L, Qian J (2017) Discrete element modeling of the effect of particle size distribution on the small strain stiffness of granular soils. Particuology 32:21-29. https://doi.org/10.1016/j.partic.2016.08.002

17. Wiącek J, Molenda M (2018) Numerical analysis of compression mechanics of highly polydisperse granular mixtures with different PSD-s. Granul Matter 20:17. https://doi.org/10.1007/s10035-018-0788-z 
18. Yan WM, Dong J (2011) Effect of Particle Grading on the Response of an Idealized Granular Assemblage. Int $\mathrm{J}$ Geomech 11:276-285. https://doi.org/10.1061/(ASCE)GM.1943-5622.0000085

19. Azéma E, Linero S, Estrada N, Lizcano A (2017) Shear strength and microstructure of polydisperse packings: The effect of size span and shape of particle size distribution. Phys Rev E 96:022902. https://doi.org/10.1103/PhysRevE.96.022902

20. Voivret C, Radjaï F, Delenne J-Y, El Youssoufi MS (2007) Space-filling properties of polydisperse granular media. Phys Rev E 76:021301. https://doi.org/10.1103/PhysRevE.76.021301

21. Kozicki J, Donzé FV (2008) A new open-source software developed for numerical simulations using discrete modeling methods. Comput Methods Appl Mech Eng 197:4429-4443. https://doi.org/10.1016/j.cma.2008.05.023

22. Cundall PA, Hart RD (1992) NUMERICAL MODELLING OF DISCONTINUA. Eng Comput 9:101-113. https://doi.org/10.1108/eb023851

23. Coetzee CJ (2017) Review: Calibration of the discrete element method. Powder Technol 310:104-142. https://doi.org/10.1016/j.powtec.2017.01.015

24. Marczewska I, Rojek J, Kačianauskas R (2016) Investigation of the effective elastic parameters in the discrete element model of granular material by the triaxial compression test. Arch Civ Mech Eng 16:64-75. https://doi.org/10.1016/j.acme.2015.09.010

25. Gu X, Huang M, Qian J (2014) DEM investigation on the evolution of microstructure in granular soils under shearing. Granul Matter 16:91-106. https://doi.org/10.1007/s10035-013-0467-z

26. Guo N, Zhao J (2013) The signature of shear-induced anisotropy in granular media. Comput Geotech 47:1-15. https://doi.org/10.1016/j.compgeo.2012.07.002

27. Katagiri J, Matsushima T, Yamada Y (2010) Simple shear simulation of 3D irregularly-shaped particles by image-based DEM. Granul Matter 12:491-497. https://doi.org/10.1007/s10035-010-0207-6

28. Goldenberg C, Goldhirsch I (2005) Friction enhances elasticity in granular solids. Nature 435:188-191. https://doi.org/10.1038/nature03497

29. Cole DM (2015) Laboratory observations of frictional sliding of individual contacts in geologic materials. Granul Matter 17:95-110. https://doi.org/10.1007/s10035-014-0526-0

30. Cheung G, O'Sullivan C (2008) Effective simulation of flexible lateral boundaries in two- and three-dimensional DEM simulations. Particuology 6:483-500. https://doi.org/10.1016/j.partic.2008.07.018 
31. Thornton C (2000) Numerical simulations of deviatoric shear deformation of granular media. Géotechnique

50:43-53. https://doi.org/10.1680/geot.2000.50.1.43

32. Aboul Hosn R, Sibille L, Benahmed N, Chareyre B (2017) Discrete numerical modeling of loose soil with spherical particles and interparticle rolling friction. Granul Matter 19:4. https://doi.org/10.1007/s10035-016-0687-0

33. Tong A-T, Catalano E, Chareyre B (2012) Pore-Scale Flow Simulations: Model Predictions Compared with Experiments on Bi-Dispersed Granular Assemblies. Oil Gas Sci Technol - Rev D’IFP Energ Nouv 67:743-752. https://doi.org/10.2516/ogst/2012032

34. Head KH (1994) Manual of soil laboratory testing. Vol. 2: Permeability, shear strength and compressibility tests, 2 . ed. Wiley, Chichester

35. Nader F, Silvani C, Djeran-Maigre I (2017) Grain breakage under uniaxial compression using a three-dimensional discrete element method. Granul Matter 19:53. https://doi.org/10.1007/s10035-017-0737-2

36. Huang X, Hanley KJ, O'Sullivan C, Kwok FCY (2014) Effect of sample size on the response of DEM samples with a realistic grading. Particuology 15:107-115. https://doi.org/10.1016/j.partic.2013.07.006

37. da Cruz F, Emam S, Prochnow M, et al (2005) Rheophysics of dense granular materials: Discrete simulation of plane shear flows. Phys Rev E 72:021309. https://doi.org/10.1103/PhysRevE.72.021309

38. Lopera Perez JC, Kwok CY, O'Sullivan C, et al (2016) Assessing the quasi-static conditions for shearing in granular media within the critical state soil mechanics framework. Soils Found 56:152-159. https://doi.org/10.1016/j.sandf.2016.01.013

39. Love AEH (2013) A treatise on the mathematical theory of elasticity, 4. ed. Cambridge Univ. Press, Cambridge

40. Weber J (1996) Recherches concernant les contraintes intergranulaires dans les milieux pulvérulents. Bull Liaison Ponts--Chaussées 20:1-20

41. Itasca Consulting Group (2007) PFC3D Version 4.0 User Manual. Itasca Consulting Group, Minneapolis, MN, USA

42. Zhao T, Dai F, Xu NW, et al (2015) A composite particle model for non-spherical particles in DEM simulations. Granul Matter 17:763-774. https://doi.org/10.1007/s10035-015-0596-7

43. Amirpour Harehdasht S, Hussien MN, Karray M, et al (2019) Influence of particle size and gradation on shear strength-dilation relation of granular materials. Can Geotech J 56:208-227. https://doi.org/10.1139/cgj-2017-0468

44. Salot C, Gotteland P, Villard P (2009) Influence of relative density on granular materials behavior: DEM simulations of triaxial tests. Granul Matter 11:221-236. https://doi.org/10.1007/s10035-009-0138-2 
45. Yoshimoto N, Wu Y, Hyodo M, Nakata Y (2016) Effect of relative density on the shear behaviour of granulated coal ash. Geomech Eng 10:207-224. https://doi.org/10.12989/GAE.2016.10.2.207

46. Ovalle C, Bard E, Dano C, et al (2015) A Review of Large Triaxial Tests on Coarse Rockfill Samples. In: Manzanal D, Sfriso AO (eds). IOS Press, Buenos Aires, Argentina, pp 3256-3263

47. Wichtmann T, Kimmig I, Triantafyllidis T (2017) On correlations between "dynamic" (small-strain) and "static" (large-strain) stiffness moduli - An experimental investigation on 19 sands and gravels. Soil Dyn Earthq Eng 98:7283. https://doi.org/10.1016/j.soildyn.2017.03.032

48. Hamidi B, Varaksin S, Nikraz H (2013) Relative density concept is not a reliable criterion. Proc Inst Civ Eng - Ground Improv 166:78-85. https://doi.org/10.1680/grim.11.00014

49. Tavenas F, Rochelle PL (1972) Accuracy of relative density measurements. Géotechnique 22:549-562. https://doi.org/10.1680/geot.1972.22.4.549

50. Ha Giang PH, Van Impe PO, Van Impe WF, et al (2017) Small-strain shear modulus of calcareous sand and its dependence on particle characteristics and gradation. Soil Dyn Earthq Eng 100:371-379. https://doi.org/10.1016/j.soildyn.2017.06.016

51. Liu X, Yang J (2018) Influence of size disparity on small-strain shear modulus of sand-fines mixtures. Soil Dyn Earthq Eng 115:217-224. https://doi.org/10.1016/j.soildyn.2018.08.011

52. Hardin BO, Black WL (1966) Sand Stiffness Under Various Triaxial Stresses. J Soil Mech Found Div 92:27-42. https://doi.org/10.1061/JSFEAQ.0000865

53. Hardin BO, Richart FE (1963) Elastic Wave Velocities in Granular Soils. J Soil Mech Found Div 89:33-65. https://doi.org/10.1061/JSFEAQ.0000493

54. Lo Presti DCF, Jamiolkowski M, Pallara O, et al (1997) Shear modulus and damping of soils. Géotechnique 47:603-617. https://doi.org/10.1680/geot.1997.47.3.603

55. Taiebat M, Mutabaruka P, Pellenq R, Radjai F (2017) Effect of particle size distribution on 3D packings of spherical particles. EPJ Web Conf 140:02030. https://doi.org/10.1051/epjconf/201714002030 
Table 1 Parameters of the DEM model

\begin{tabular}{ll}
\hline Parameter & Value \\
\hline Particle density $\rho\left(\mathrm{kg} / \mathrm{m}^{3}\right)$ & 2,650 \\
Inter-particle friction $\mu$ & 0.7 \\
Young's modulus $E_{c}(\mathrm{~Pa})$ & $10^{9}$ \\
Particle stiffness ratio $\theta$ & 0.7 \\
Damping ratio $\alpha$ & 0.2 \\
\hline
\end{tabular}

638 
Table 2 Information for DEM samples simulated in this study

\begin{tabular}{cccccccc}
\hline $\begin{array}{c}\text { Sample } \\
\text { No. }\end{array}$ & $s$ & $b$ & $e_{0}^{\max }$ & $e_{0}^{\min }$ & $C_{u}$ & $\gamma$ & $I_{G}$ \\
\hline 1 & 0.40 & 0.1 & 0.688 & 0.556 & 1.10 & 0.118 & 0.047 \\
2 & 0.40 & 0.5 & 0.692 & 0.539 & 1.38 & 0.204 & 0.082 \\
3 & 0.40 & 1.0 & 0.679 & 0.529 & 1.43 & 0.243 & 0.097 \\
4 & 0.40 & 2.0 & 0.678 & 0.527 & 1.39 & 0.245 & 0.098 \\
5 & 0.40 & 4.0 & 0.678 & 0.534 & 1.26 & 0.204 & 0.081 \\
6 & 0.54 & 0.5 & 0.673 & 0.523 & 1.51 & 0.207 & 0.112 \\
7 & 0.54 & 1.0 & 0.652 & 0.505 & 1.62 & 0.238 & 0.129 \\
8 & 0.54 & 2.0 & 0.647 & 0.505 & 1.60 & 0.231 & 0.125 \\
9 & 0.54 & 4.0 & 0.656 & 0.515 & 1.42 & 0.194 & 0.105 \\
10 & 0.89 & 0.5 & 0.602 & 0.468 & 1.86 & 0.213 & 0.189 \\
\hline
\end{tabular}

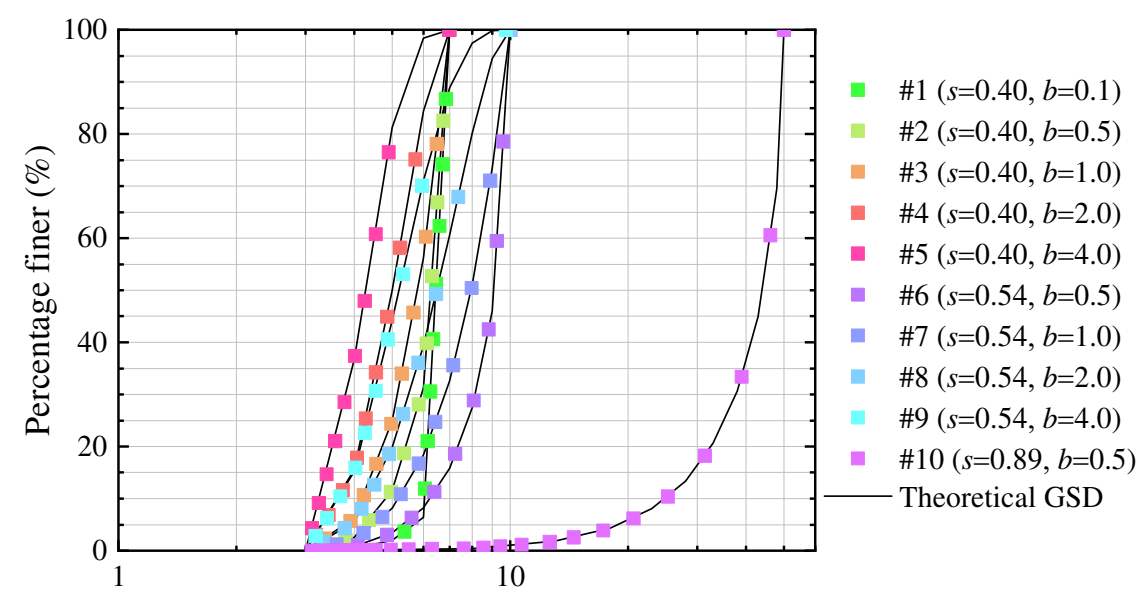

Diameter (mm)

Fig. 1 Theoretical (solid lines) and generated (symbols) GSDs of numerical samples. The values of $s$ and $b$ are given 642 in the parentheses behind sample labels 

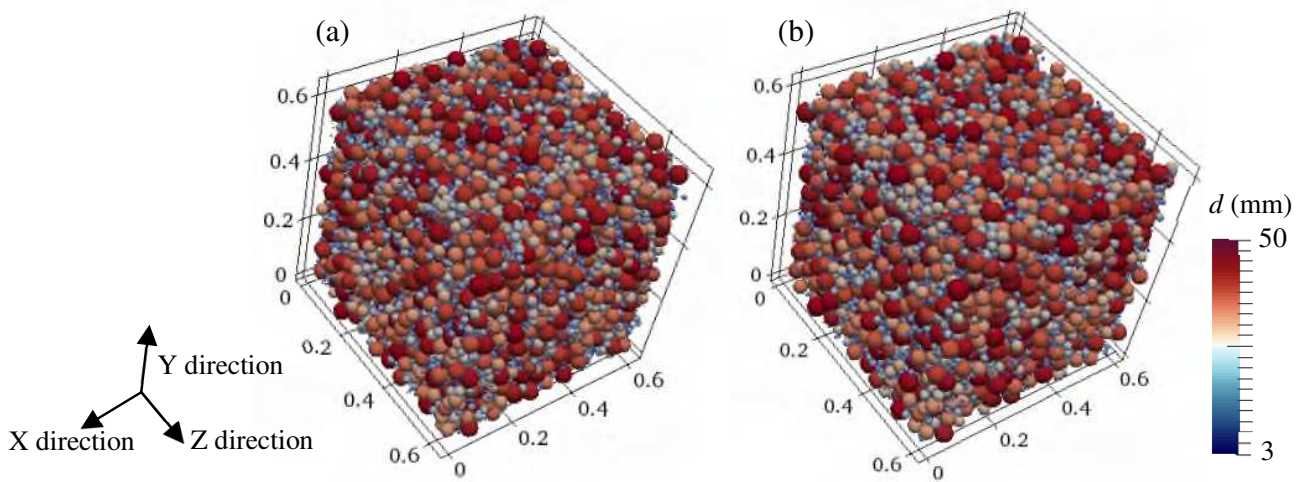

645

Fig. 2 Numerical samples generated according to the GSD $(s=0.89$ and $b=0.5)$ at the (a) loose and (b) dense states.

646

The dimensions of the samples can be approximately identified through the tick labels in meters. The particles are 647 color coded according to their sizes 


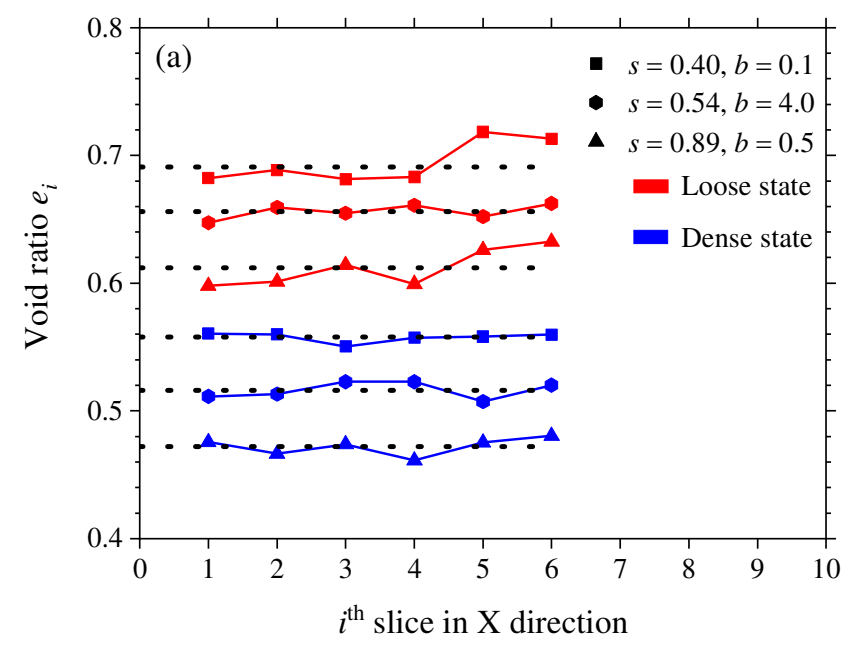

649

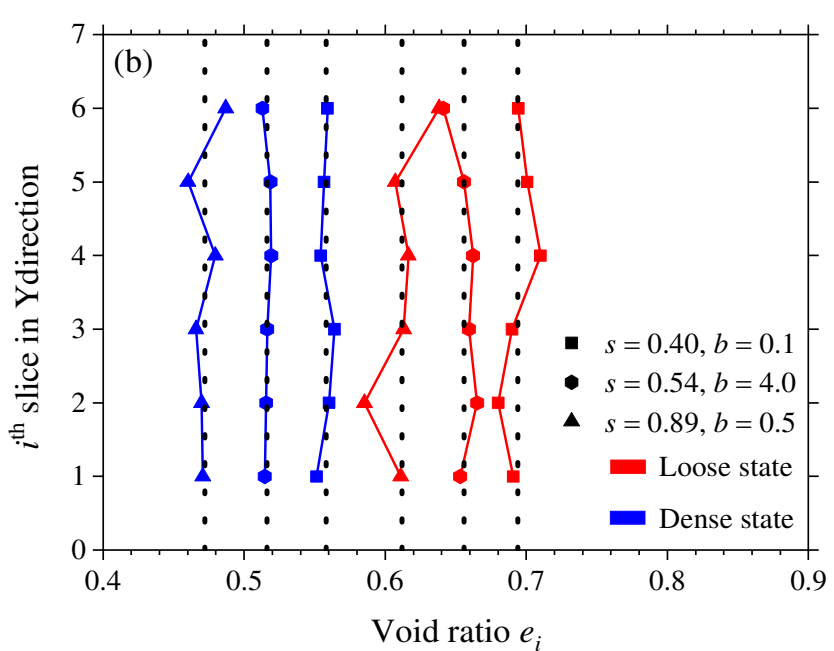

650

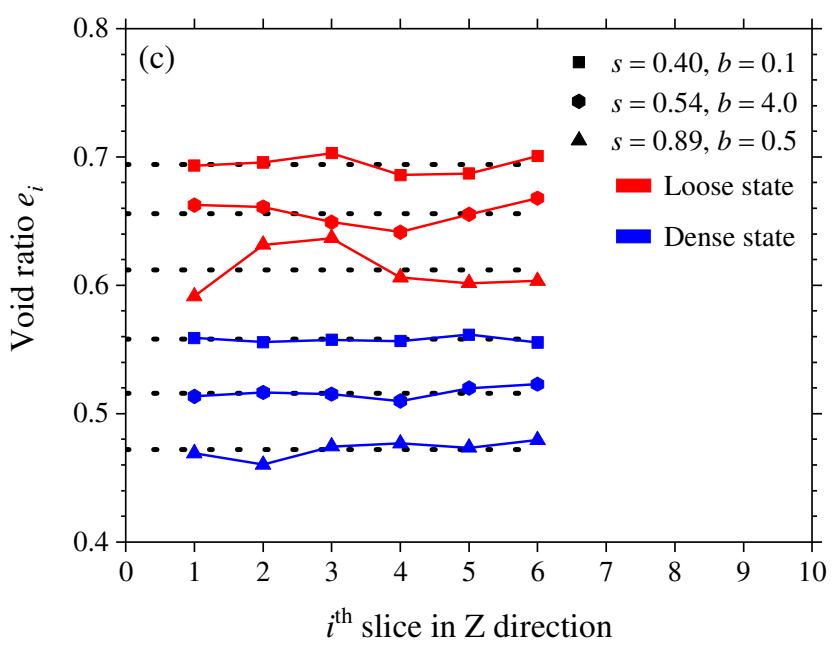

Fig. 3 Distributions of void ratio in (a) X, (b) $\mathrm{Y}$ and (c) Z directions for six samples with various GSDs at different density states. The dot lines indicate the void ratio of the whole sample 

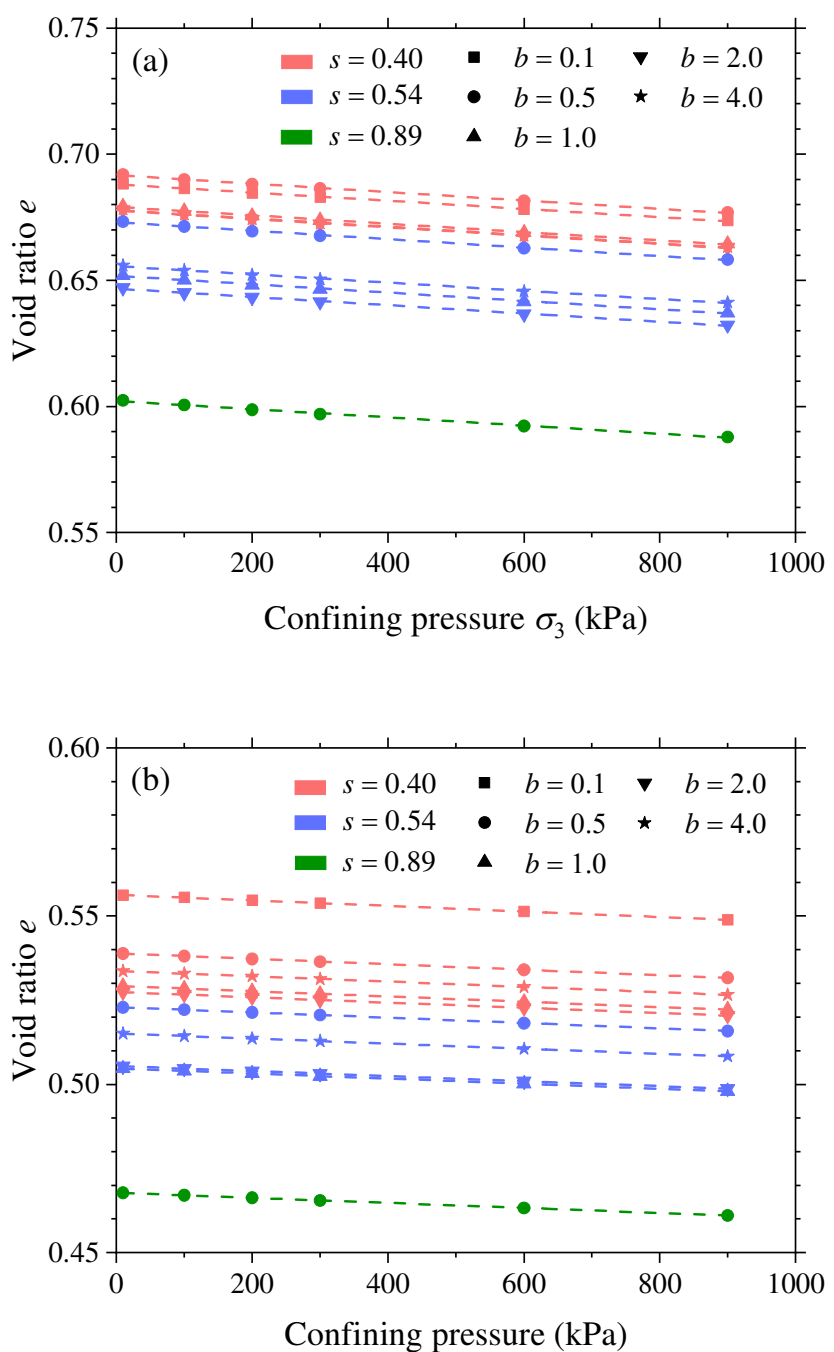


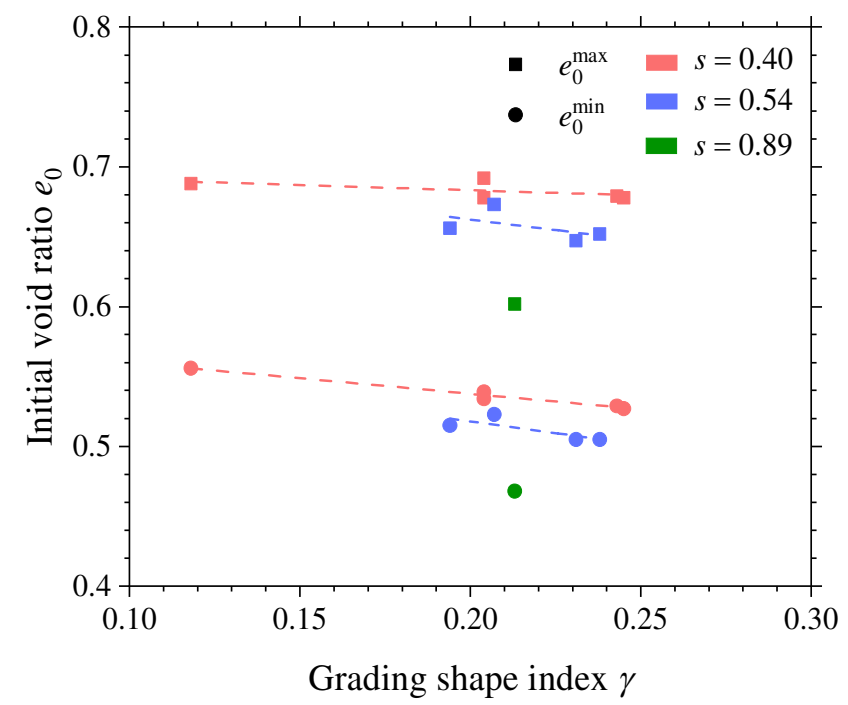

660

Fig. 5 The effect of the size span and the shape index of GSD on the initial void ratio $e_{0}$ of samples at two density 661 states

662 


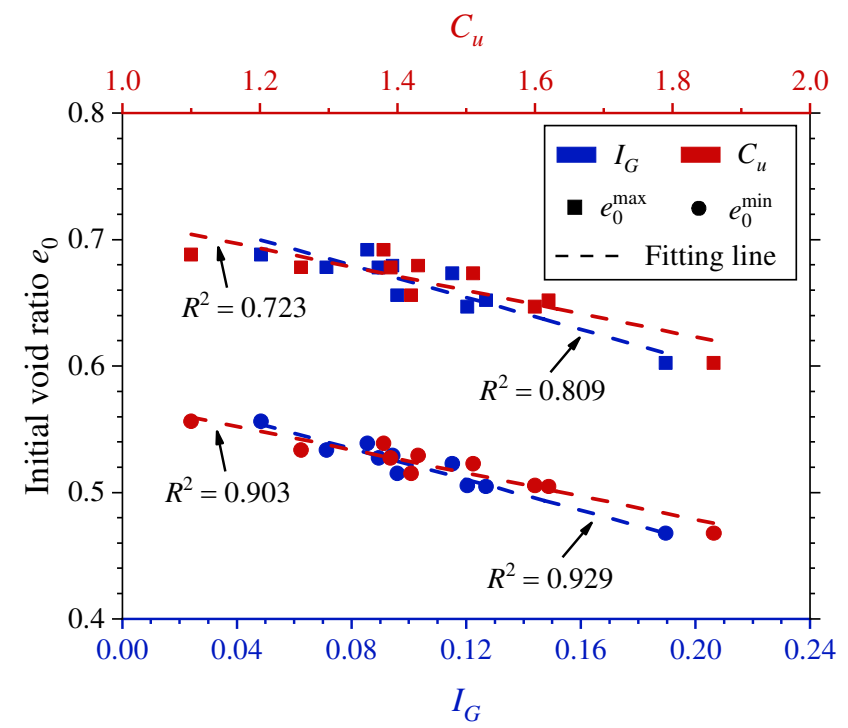

663

Fig. 6 Relationship between the initial void ratio $e_{0}$ and different measures of size polydispersity in present study 665 


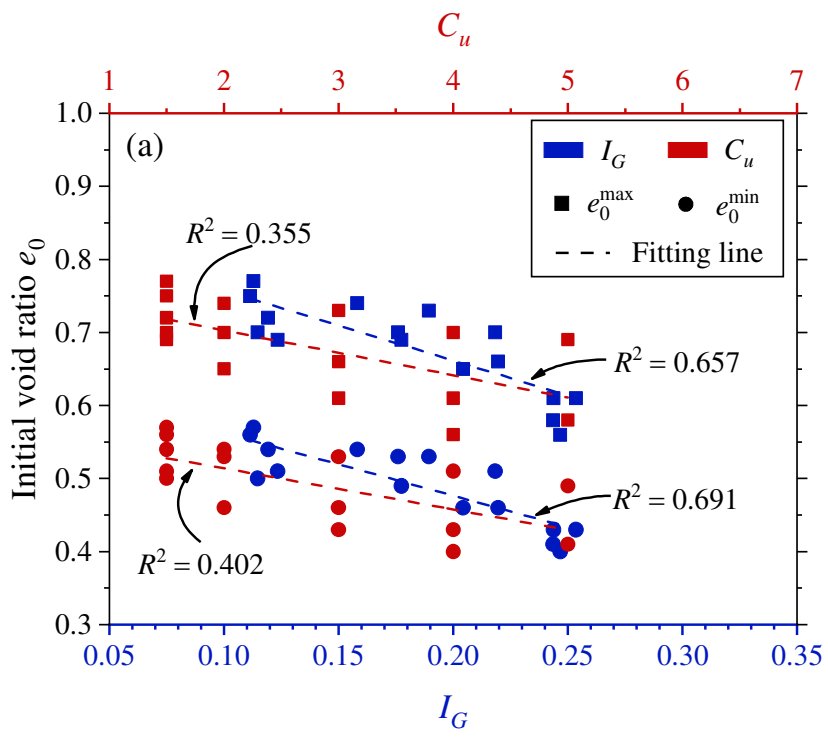

666

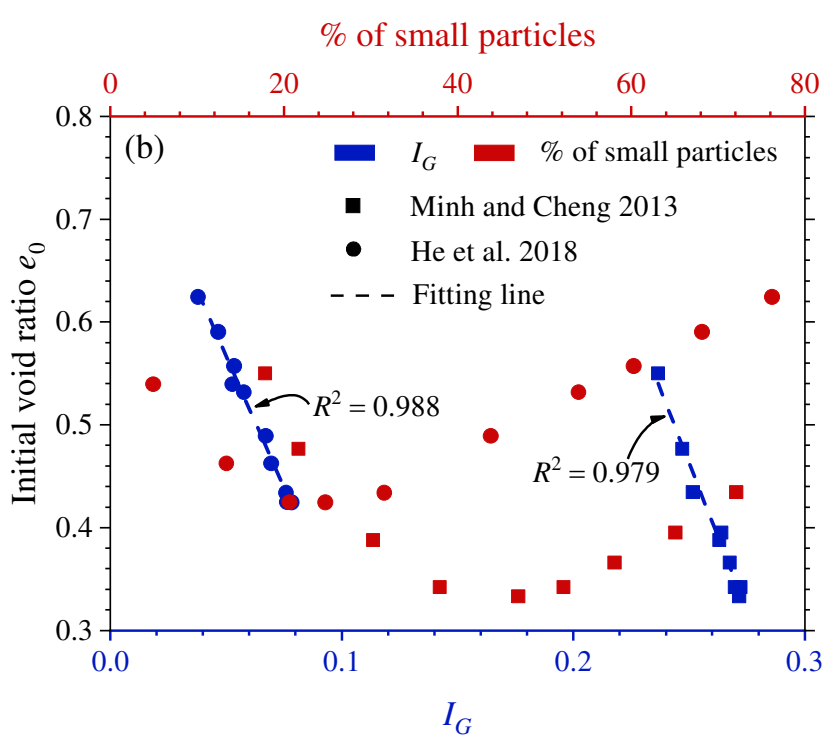

667

Fig. 7 Relation of the initial void ratio $e_{0}$ with various measures of size polydispersity from previous (a) experimental 669 [43] and (b) numerical $[9,10]$ data

670 

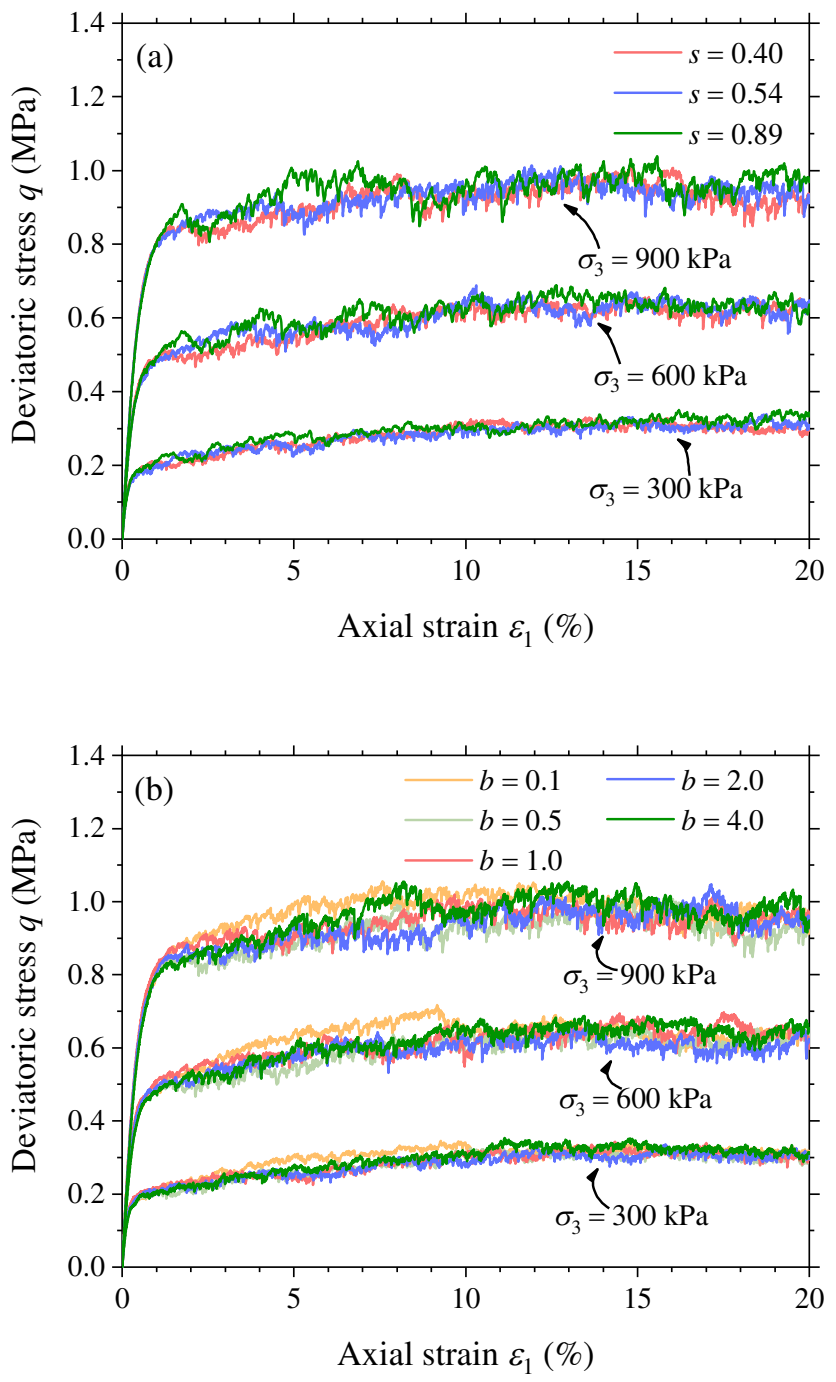

Fig. 8 Stress-strain response of loose samples with various GSDs for (a) $b=0.5$ and (b) $s=0.40$ under different confining pressures 

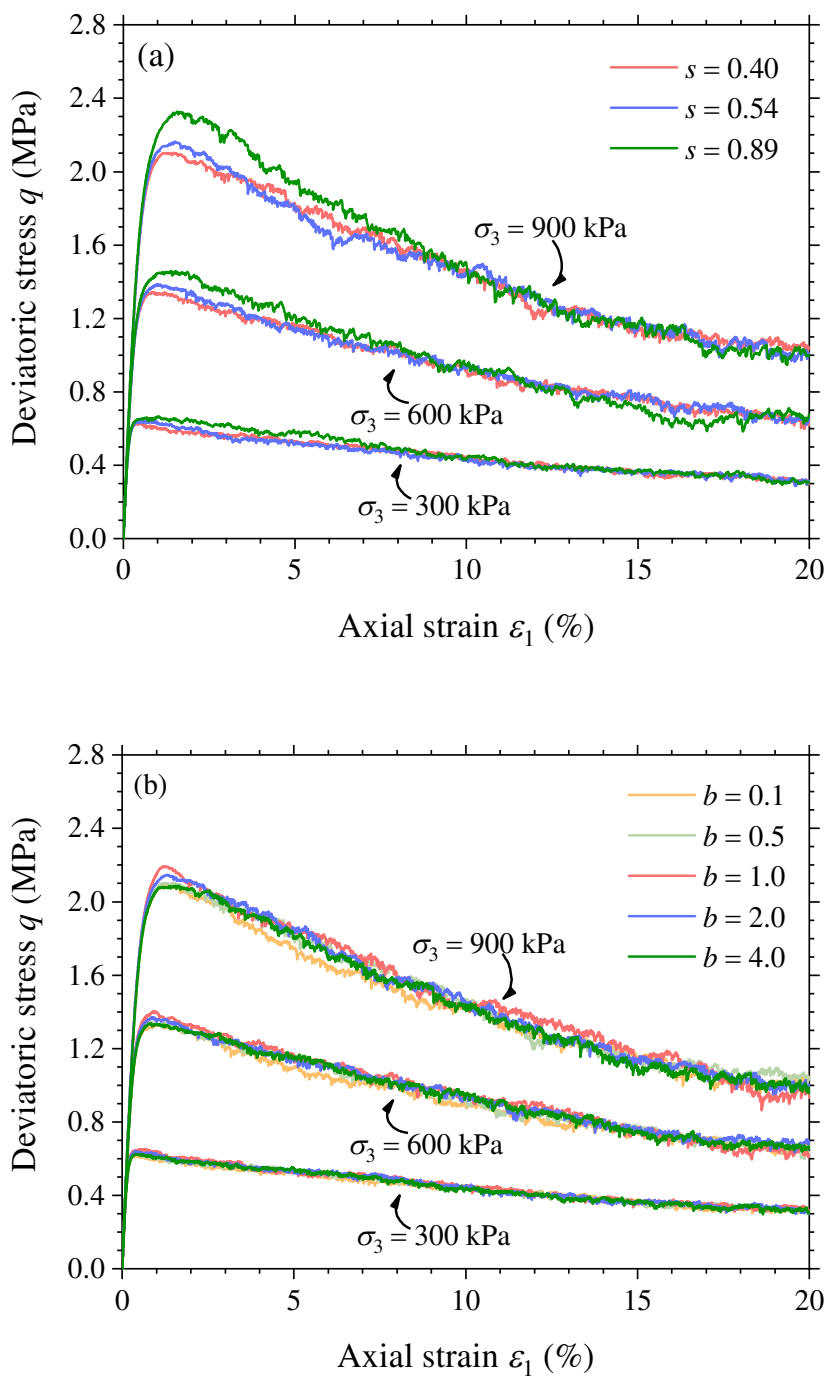

Fig. 9 Stress-strain response of dense samples with various GSDs for (a) $b=0.5$ and (b) $s=0.40$ under different confining pressures 


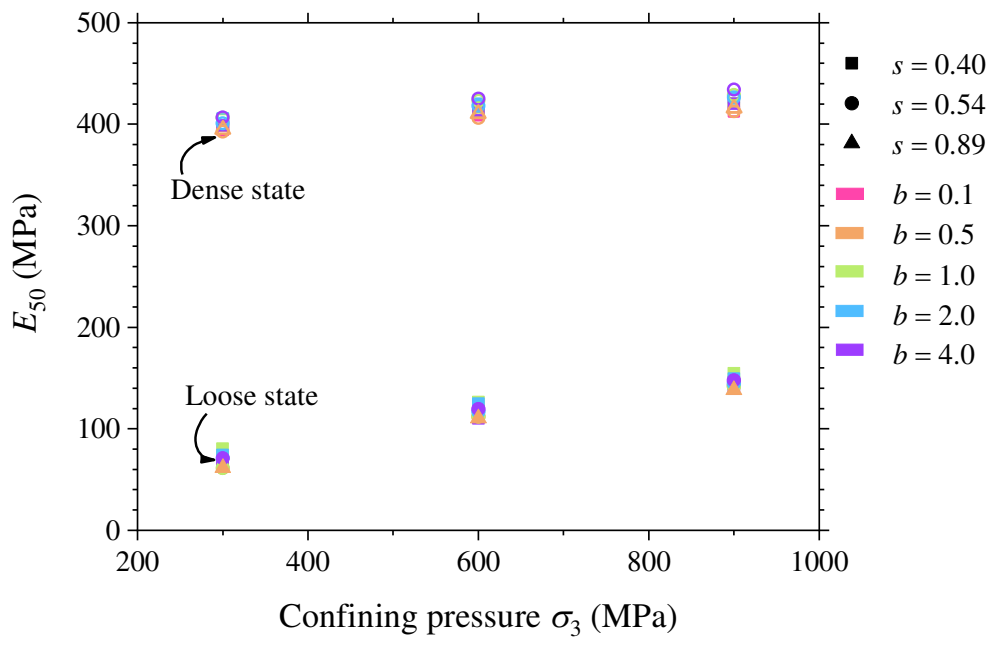

682 Fig. 10 The relationship between the deformation modulus $\left(E_{50}\right)$ and confining pressure $\left(\sigma_{3}\right)$ for samples with various 683 GSDs at two density states 

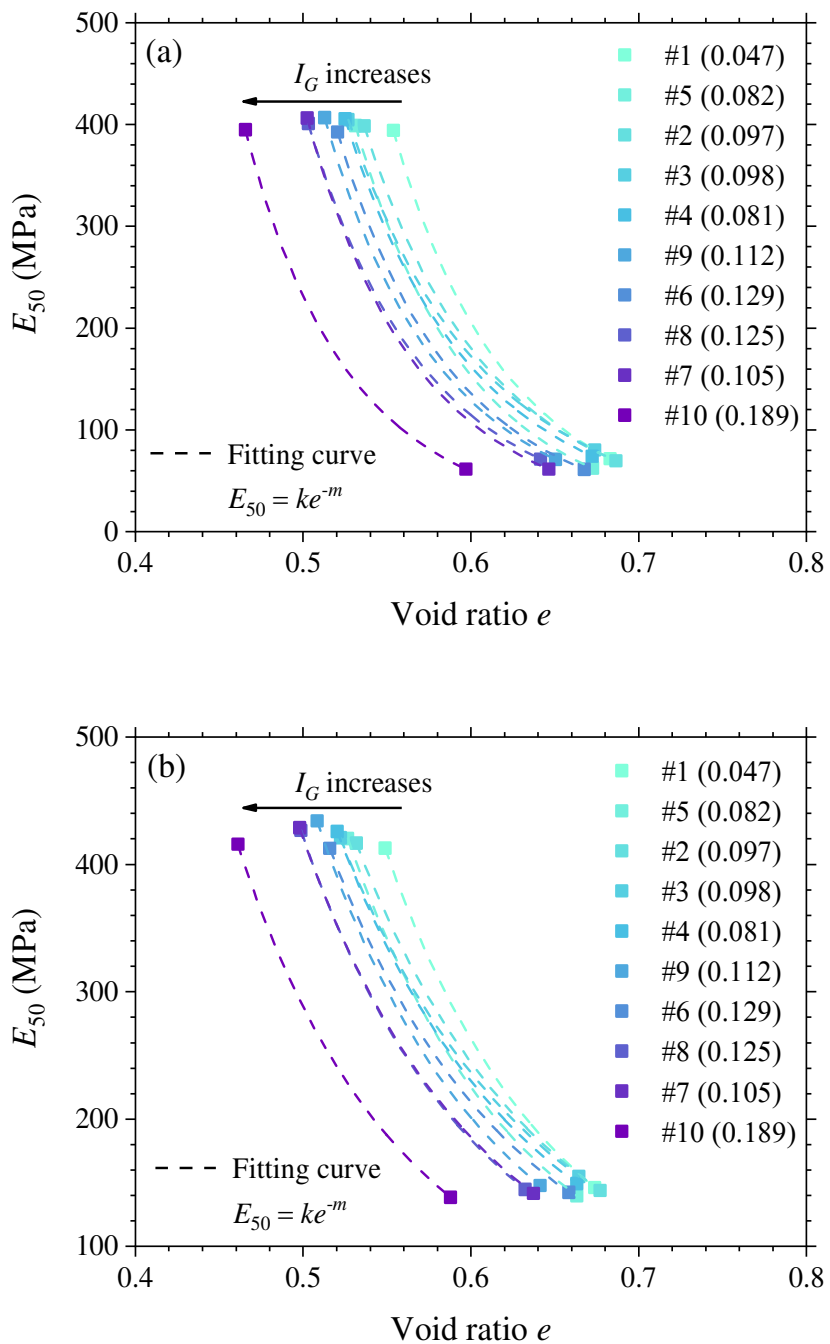

687 Fig. 11 The relationship between deformation modulus $\left(E_{50}\right)$ and void ratio $(e)$ at (a) $300 \mathrm{kPa}$ and (b) $900 \mathrm{kPa}$ confining pressures for samples with various GSDs. The values of $I_{G}$ are given in the bracket behind a sample label. 689 The data markers are dyed from light to dark as $I_{G}$ increases 


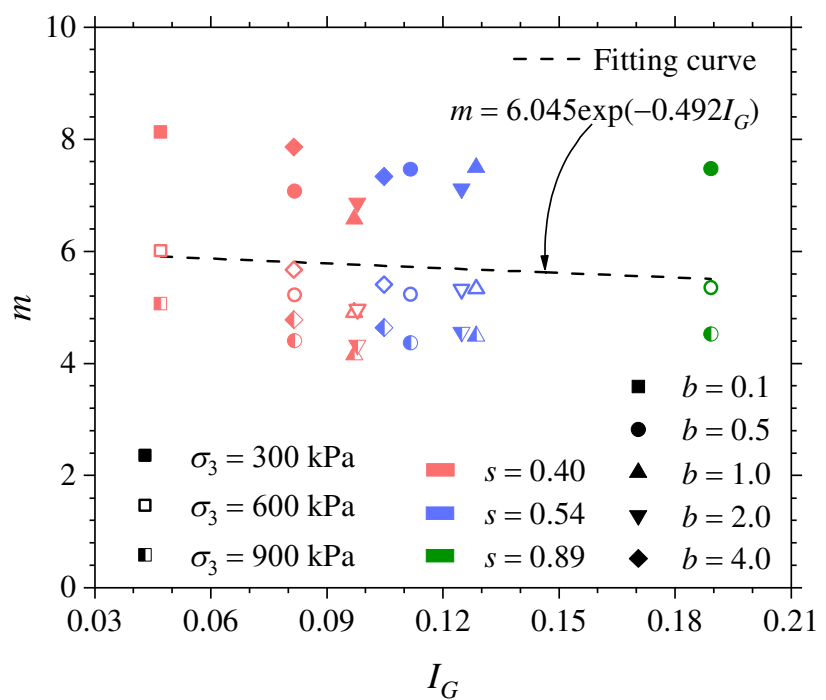

691

692

Fig. 12 Variation of parameter $m$ with $I_{G}$

693 


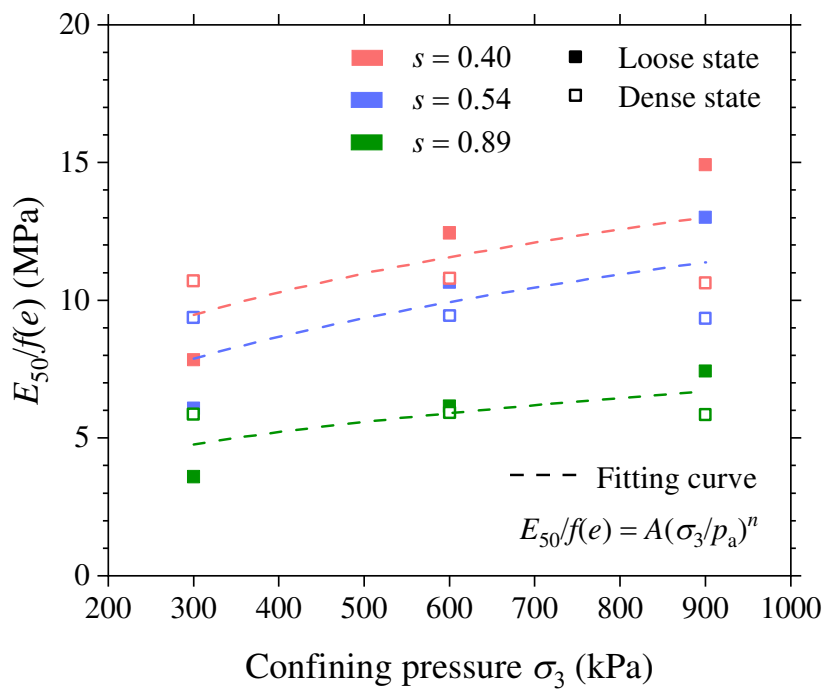

695 Fig. 13 Void ratio-normalized $E_{50}$ versus confining pressure $\left(\sigma_{3}\right)$ for three samples with grading parameter $b=0.5$.

696 The $E_{50} / f(e)$ data from samples at the loose and dense states are denoted by solid and open symbols, respectively 


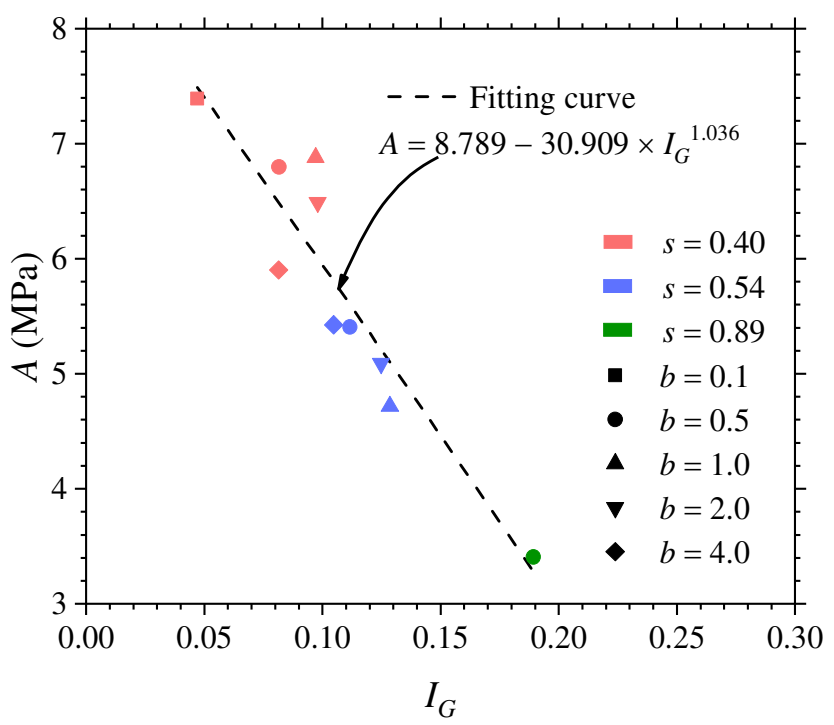

698

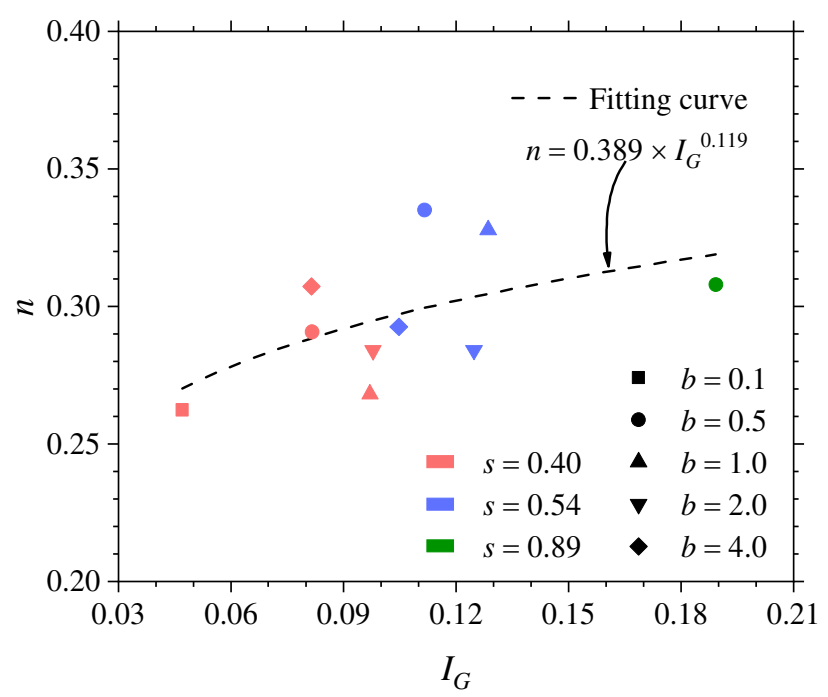

699

Fig. 14 Parameters (a) $A$ and (b) $n$ in dependence of $I_{G}$ 

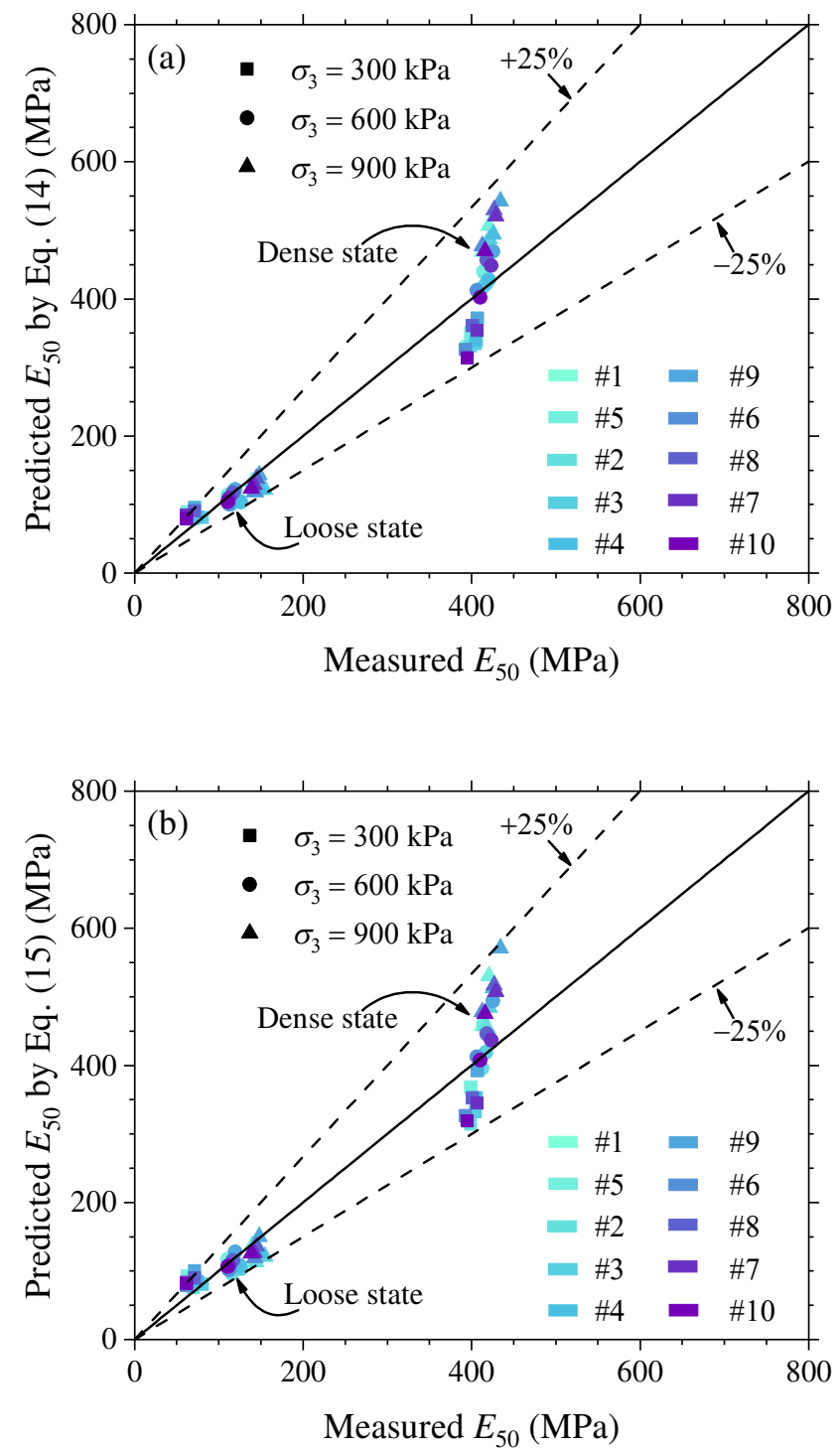

704 Fig. 15 Measured against predicted $E_{50}$ using (a) Eq. (14) and (b) Eq. (15). The data markers are dyed from light to dark as $I_{G}$ increases 


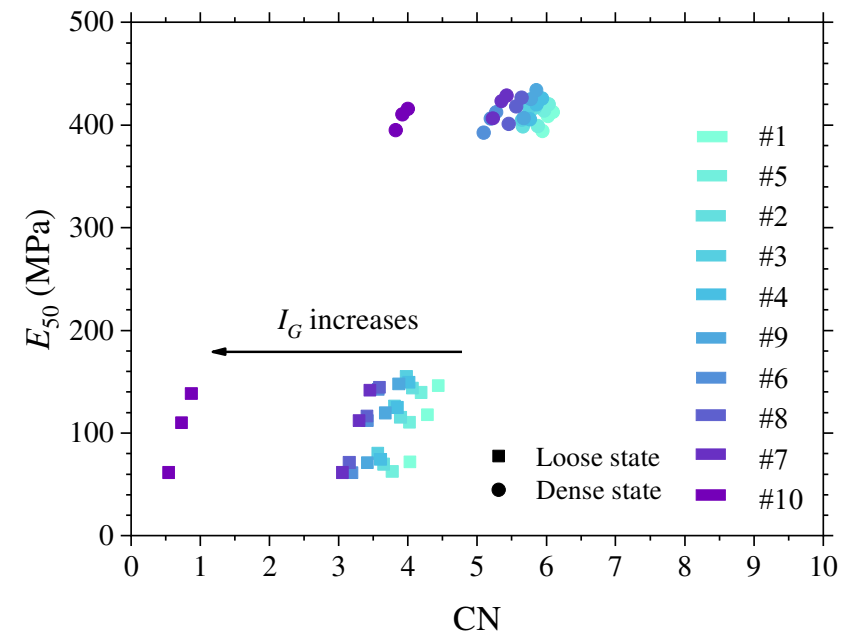

707

Fig. 16 Correlation between the deformation modulus $\left(E_{50}\right)$ and the average coordination number $(\mathrm{CN})$ of different samples before shearing. The data markers are dyed from light to dark as $I_{G}$ increases 


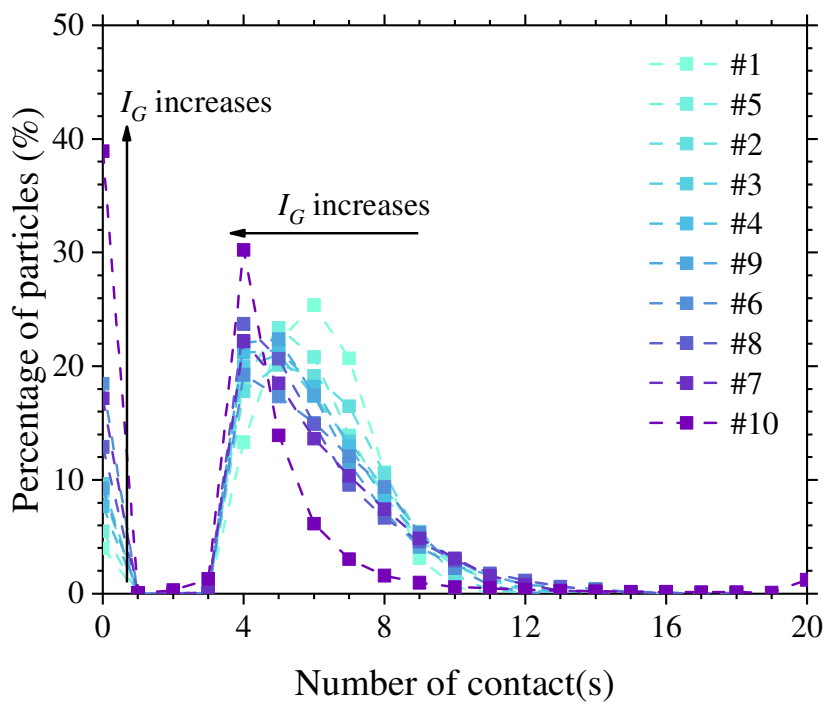

712 Fig. 17 Distributions of the number of contact(s) for particles of dense samples with various GSDs. The samples before triaxial compression loading are examined and the data markers are dyed from light to dark as $I_{G}$ increases 


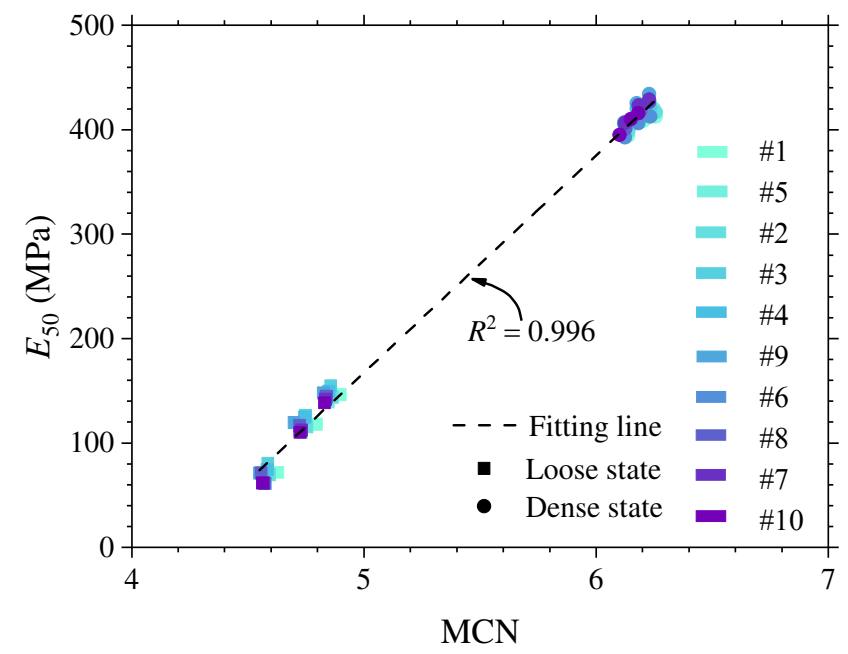

716 Fig. 18 Correlation between the deformation modulus $\left(E_{50}\right)$ and the mechanical average coordination number $(\mathrm{MCN})$ of different samples before shearing. The data markers are dyed from light to dark as $I_{G}$ increases 

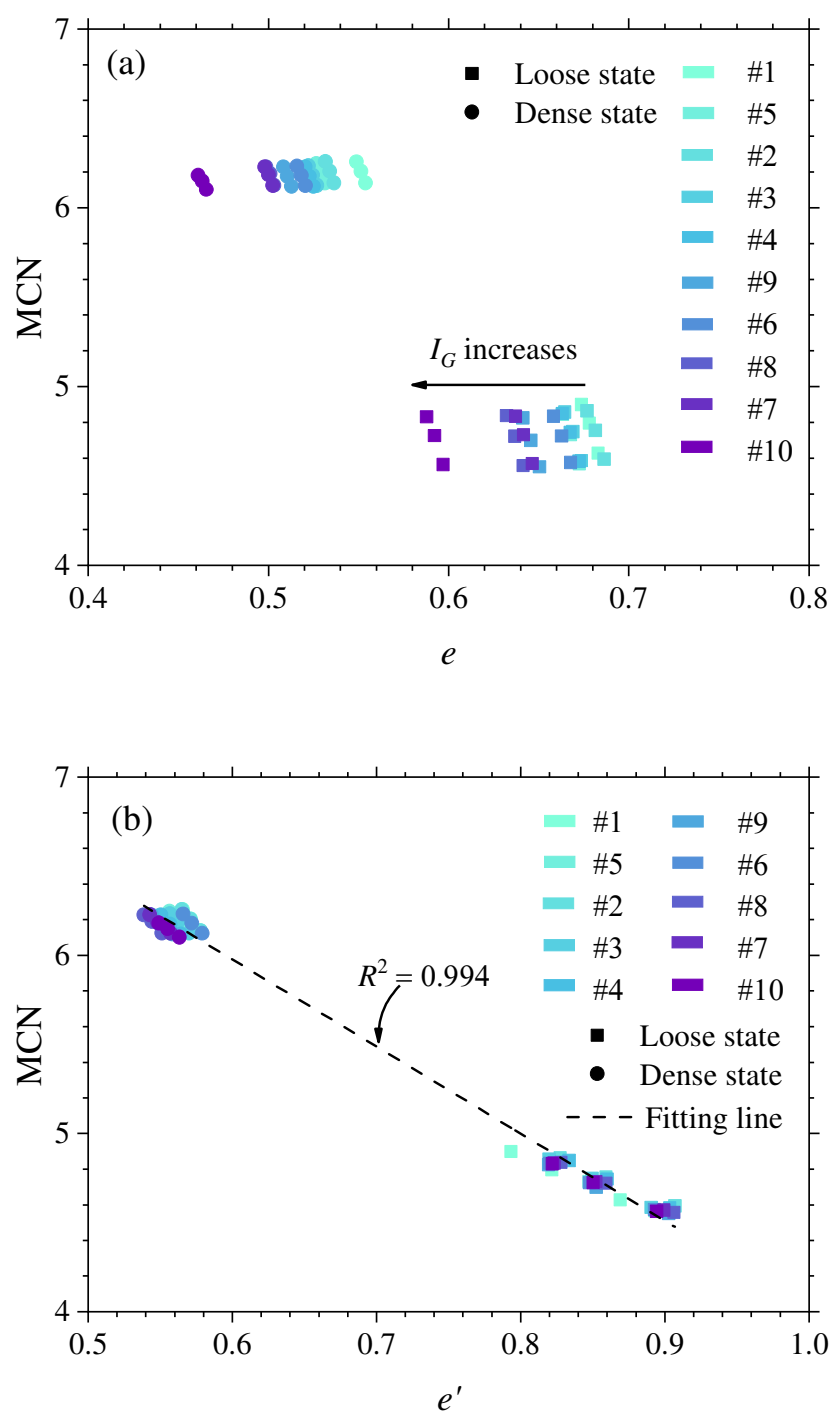

Fig. 19 Correlations of the mechanical average coordination number (MCN) with (a) the void ratio (e) and (b) the effective void ratio $\left(e^{\prime}\right)$ of different samples before shearing. The data markers are dyed from light to dark as $I_{G}$ increases 